\title{
Graphene-based two-dimensional Janus materials
}

Sze-Wing $\mathrm{Ng}^{1}$, Nuruzzaman Noor $^{1}$ and Zijian Zheng (D) $^{1}$

\begin{abstract}
Two-dimensional (2D) Janus materials with opposing components and properties on two sides have recently attracted fevered attention from various research fields for use as, for example, oil/water separating membranes, interfacial layers for mass transfer, 2D sensors and actuators. The Janus structure allows for a unidirectional transportation system and programmed response to certain stimuli to be achieved. Graphene, the 2D honeycomb network formed from one atomic layer of carbon atoms, has also received substantial research interest because of its intriguing structure and fascinating properties. The high mechanical strength, flexibility and optical transparency make graphene a unique candidate as a building block of 2D Janus materials through asymmetric modification with different functional groups on the graphene surfaces. This article reviews graphene-based 2D Janus materials, starting with a theoretical understanding of the behavior of Janus graphene. Then, different strategies for fabricating Janus graphene and its derivatives are reviewed in detail according to the chemical strategies of the modification methods. The applications of graphene-based Janus materials are discussed with a specific focus on the Janus structures that lead to bandgap engineering, as well as the construction of a responsive system on graphene.
\end{abstract}

\section{Introduction to two-dimensional Janus materials}

In Roman mythology, Janus was the God of beginnings, ends and transitions and had two faces to better survey both the past and the future ${ }^{1}$. Currently, the word "Janus" has been adopted by the scientific materials community to denote materials that show different properties on two opposing sides or faces. The term "Janus materials" was first adopted by C. Casagrande in 1988 to describe a spherical glass bead with asymmetric facial hydrophilicity $^{2}$, and intensive interest on Janus materials was aroused from the public in 1991, after Pierre-Gilles de Gennes gave his Nobel lecture on the concept of the "Janus Grain". Over the past 30 years, a large number of Janus materials have been developed that have led to a wide range of new applications in macro/micro-structures ${ }^{3,4}$, electronic thin films ${ }^{5-8}$, sensors 9 , surfactants ${ }^{10}$, oil/water separation membranes ${ }^{11-14}$,

Correspondence: Zijian Zheng (zijian.zheng@polyu.edu.hk)

${ }^{1}$ Laboratory for Advanced Interfacial Materials and Devices, Institute of Textiles and Clothing, The Hong Kong Polytechnic University, Hung Hom, Hong Kong SAR, China cell and drug encapsulations ${ }^{15,16}$ and biomimetic interfaces ${ }^{3,17-19}$.

Among the many Janus structures ${ }^{3,4,10,20,21}$, twodimensional (2D) Janus materials in the form of ultrathin sheets, thin films and discs have received rapidly increasing attention in recent years. The lotus leaf structure is a typical example of a 2D Janus material existing in nature: its upper side is superhydrophobic (waterrepellent), while the lower side is superoleophobic (fat-repellent), making the water freely roll atop of the leaves ${ }^{18,19}$. Conventionally, 2D Janus materials were prepared by bottom-up methods, including crosslinking of self-assembled block copolymers ${ }^{22}$, selective chemical functionalization of freestanding nanomembranes ${ }^{23}$ and layer-by-layer self-assembly at air-water interfaces ${ }^{24}$, or by top-down methods such as multi-step etching of a wafer substrate ${ }^{25}$ and colloid milling of Janus hollow spheres $^{26-30}$. However, it is difficult to make ultrathin 2D Janus materials at the nanoscale with precise control of size, morphology and surface properties by using such methods. 
It was not until 2004 that researchers found better ways to prepare 2D Janus nanomaterials using graphene, which is a $2 \mathrm{D}$ single-atom-thick honeycomb lattice made up of carbon atoms. In 2004, Geim and Novoselov ${ }^{31}$ reported the intentional empirical discovery of isolated monolayer graphene through the mechanical exfoliation of graphite. The monolayer graphene sheet was found to possess ultrahigh mechanical flexibility and durability (Young's modulus: $\sim 1 \mathrm{TPa}$; intrinsic tensile strength: $130 \mathrm{GPa})^{32}$, superb optical transparency ( $\sim 97 \%$ transmittance to visible and infrared light), high thermal conductivity $(\sim 5000$ $\left.\mathrm{W} \mathrm{mK}{ }^{-1}\right)$, high stability to air and moisture below $300^{\circ} \mathrm{C}$ and extremely high charge carrier mobility $\left(\sim 100,000 \mathrm{~cm}^{2}\right.$ $\mathrm{V}^{-1} \mathrm{~s}^{-1}$ ) at room temperature. Importantly, graphene and its derivatives, such as graphene nanoflakes, graphene oxide (GO) and reduced graphene oxide (rGO), can be readily synthesized via scalable methods, including chemical vapor deposition (CVD) $)^{33-35}$ and various liquidphase exfoliation methods ${ }^{36,37}$. In recent years, scientists have made use of graphene as a building block for fabricating various nano-, micro- and macro-scale 2D Janus materials through selective surface modification approaches. These Janus modification strategies have significantly expanded the potential of graphene and its derivatives into a wider range of applications including batteries and energy storage ${ }^{38-44}$, field-effect transistors $^{45-47}$, photovoltaic devices ${ }^{7,48-53}$, catalysis ${ }^{54,55}$ and sensors and actuators ${ }^{56-61}$.

In view of the rapid growth in this area, this paper aims to provide a comprehensive review of contemporary graphene-based 2D Janus materials. In this paper, we introduce the theoretical understanding of 2D Janus graphene and review the experimental methods for asymmetric functionalization of graphene and graphene derivatives. Representative examples of Janus graphene modifications towards bandgap engineering, chemical functionalization and membrane applications are given, and the new opportunities generated are also discussed. It should be noted that this article only reviews reports on the asymmetric modification of graphene and its assemblies. Reports on other modifications and applications of graphene and graphene derivatives can be found in other reviews, such as "Graphene-based composites"62, "Functionalization of Graphene: Covalent and NonCovalent Approaches, Derivatives and Applications" ${ }^{\text {"63 }}$ and "Graphene, related two-dimensional crystals, and hybrid systems for energy conversion and storage" ${ }^{\text {"64 }}$.

\section{Theoretical understanding of Janus graphene}

The attention on graphene-based Janus materials in the research community originates from the theoretical understanding of graphene's atomic modification. Monolayer graphene is a zero-bandgap semiconductor. For insight into widening the bandgap in graphene, a significant number of computational calculations have been carried out on graphene hydrogenation and halogenation to study the band structure engineering possibilities. In 2007 , Sofo et al. ${ }^{65}$ predicted a graphene derivative named "graphane" via density functional theory (DFT). Graphane was suggested to possess a fully saturated hydrocarbon structure constructed by bonding $\mathrm{H}$ atoms on both sides of a single graphene layer, leading to bandgap widening $\left(E_{\mathrm{g}}=3.5 \mathrm{eV}\right.$ for the chair conformer, and $E_{\mathrm{g}}=3.7 \mathrm{eV}$ for the boat conformer), as well as a decreased lattice constant of the $2 \mathrm{D}$ material. This pioneer work provided a strong indication that graphene can be atomically engineered to realize vastly different properties. In the following years, a great number of works have been reported on the simulation of the atomic engineering of graphene. This section reviews the works focusing on the computational study of asymmetrically engineered graphene with hydrogen and halogens.

\section{Janus graphene through hydrogenation}

In 2009, Zhou et al.$^{66}$ reported the first simulation of a single-sided hydrogenated graphene structure, named "graphone", via DFT. Constructed by removing all the bottom hydrogen atoms from graphane, graphone is a ferromagnetic semiconductor with a small indirect gap $(0.46 \mathrm{eV})$. Half-hydrogenation breaks the delocalized $\pi$-bonding network of graphene, leaving the electrons in the unhydrogenated carbon atoms localized and unpaired. The magnetic moments at these sites couple ferromagnetically with an estimated Curie temperature between 278 and $417 \mathrm{~K}$, giving rise to an infinite magnetic sheet with structural integrity and magnetic homogeneity $^{66}$. In contrast, graphane and graphene are both nonmagnetic.

By combining the cluster expansion method with DFT, Xiang et al $^{67}$ further studied the single-sided hydrogenation of graphene. They found that hydrogen atoms tend to form one-dimensional (1D) chains that lead to the formation of ripples between $\mathrm{sp}^{2}$ carbon atoms because of the competition between electronic kinetic energy and elastic strain energy. In contrast to the case of double-sided hydrogenation, phase separation between the $\mathrm{sp}^{3}$ carbon and the $\mathrm{sp}^{2}$ basal plane is prohibited by the large strain caused by the single-sided hydrogen adsorption, hence suggesting that single-sided covalent functionalization might be a better way to tune the electronic properties of graphene.

In 2012, Haberer et al. ${ }^{68}$ empirically demonstrated the first isolation of a single-sided, partially hydrogenated graphene in a " $\mathrm{C}_{4} \mathrm{H}$ " configuration through the in situ hydrogen plasma treatment of gold-supported CVD graphene. The product is expected to be a suitable material for optoelectronics in the ultraviolet (UV) range because of its wide bandgap $(3.5 \mathrm{eV})$. Based on this experimental 
work, Li et al ${ }^{69}$ further studied a number of $1 \mathrm{D}$ derivatives of the $\mathrm{C}_{4} \mathrm{H}$ structure, such as $\mathrm{C}_{4} \mathrm{H}$ nanotubes and $\mathrm{C}_{4} \mathrm{H}$ nanoribbons with DFT. The results demonstrated that a symmetric double-side hydrogenated $\mathrm{C}_{4} \mathrm{H}$ layer is impossible to achieve since the alternative double-side graphene-embedded structure is energetically preferred due to the more favorable hydrogen binding energy configurations.

\section{Janus graphene through halogenation}

Apart from hydrogenation, the halogenation reactions of graphene have also been widely studied ${ }^{70}$. Using DFT calculations, Yang et al. $^{71}$ showed that photogenerated chlorine atoms tend to adsorb onto the graphene surface to form a stable charge-transfer complex at the early stage of single-sided chlorination, leading to the p-type doping of graphene while keeping the $\mathrm{sp}^{2}$-hybridized carbon skeleton intact ${ }^{71}$. The chargetransfer complex can further undergo one of two alternative transformations: (i) adsorbed chlorine atoms can covalently bond to the para-site carbons of graphene, resulting in the formation of chlorinated graphene with a maximum coverage of $\sim 25 \%$, or (ii) two adjacent chlorine atoms combine with each other, forming chlorine molecules and subsequently desorbing from the graphene surface. The band structures of various chlorine adsorption patterns have been calculated with the bandgap of chlorinated graphene falling into a range from 0 to $1.3 \mathrm{eV}$.

\section{Combination of hydrogenation and halogenation}

Instead of single-atom modification, Singh et al. ${ }^{72}$ successfully predicted a range of hydrofluorinated graphene (HFG) structures, in which each carbon atom of graphene was covalently bonded with one $\mathrm{H}$ and one $\mathrm{F}$ atom. Depending on the configuration of hydrogen and halogen atoms, the HFG was expected to obtain a bandgap ranging from 3.34 to $5.25 \mathrm{eV}$. By using the most stable chair conformer of HGF as the model, i.e., the two sides of graphene modified with $\mathrm{H}$ or $\mathrm{F}$ atoms in a homogenous manner, $\mathrm{Li}$ et $\mathrm{al}^{73}$ noted the existence of considerable interfacial $\mathrm{C}-\mathrm{H}$... F-C hydrogen bonding among the HFG layers and reported that the stacking of two semiconducting HGF layers would yield a metallic bilayer. This kind of opposing modification with two kinds of atoms was further extended by Yang et al. ${ }^{74}$ to a combination of different halogens $(\mathrm{F}, \mathrm{Cl}$ or $\mathrm{Br}$ ) and hydrogen atoms, as shown in Fig. 1a. The results indicated that opposing sides bonding with two different elements further apart from each other in the periodic table resulted in larger band gaps.

In addition to hydrogen and halogen atoms, Ong et al. $^{75}$ calculated the configuration of Janus graphene doped with Li or K (Fig. 1b) and found that these doped
Janus graphene structures possess piezoelectric properties. Surprisingly, the same research group further predicted that piezoelectricity of monolayer graphene with opposing sides occupied by two different dopants is comparable to existing bulk piezoelectric materials, such as wurtzite, boron nitride and gallium nitride ${ }^{76}$. Such nanoscale piezoelectric materials with outstanding flexibility could play an important role in the development of soft electronics, increasing the degree of integration, as well as the energy conversion efficiency (converting mechanical energy into electric energy) of flexible devices ${ }^{77}$.

Apart from the asymmetric modifications on monolayer graphene, Yuan et al. ${ }^{78}$ reported that bilayer graphene grafted with $\mathrm{H}$ or $\mathrm{F}$ atoms on one side could form similar structures to diamondol (a bilayered structure composed of two compressed graphene monolayers-the topmost layer covered in hydroxyl groups), with repeating units denoted as $\mathrm{H}-\mathrm{C} 2$ and $\mathrm{F}-\mathrm{C} 2^{78,79}$. Based on their calculations, the $\mathrm{C}$ atoms in the first layer are fully $\mathrm{sp}^{3}$ hybridized, while only half the $C$ atoms in the second layer are $\mathrm{sp}^{3}$ hybridized; consequently, interesting ferromagnetic properties are obtained as a result of the non-hybridized states.

\section{Synthesis of Janus graphene}

The synthesis of Janus graphene strongly relies on the asymmetric modification of graphene and its derivatives, such as $\mathrm{GO} / \mathrm{rGO}$, which can be prepared by various methods, such as mechanical exfoliation, CVD, liquid exfoliation and chemical exfoliation of graphite. In the literature, the preparation methods of graphene and its derivatives have been well reviewed ${ }^{80-83}$. This section focuses on the experimental fabrication of Janus graphene, regardless of how the graphene starting materials were made.

In asymmetric modification, one side of graphene is typically protected from reaction, while the other side is exposed to atmosphere or solutions of different reagents. Thus, we categorize the preparation of Janus graphene by the use of modifying reagents including halogens and hydrogen, small organic molecules, polymers, metals and metal oxides.

\section{Asymmetric functionalization of graphene with halogens and hydrogen}

Inspired by the theoretical studies of Janus graphene discussed above, the early experimental works were largely based on the asymmetric modification of graphene through halogenation and hydrogenation. In this type of modification, the bare graphene is made via mechanical exfoliation or CVD. The upper side of this single or fewlayer graphene is exposed to gaseous reactive reagents, while the bottom side is protected. 
a)

i) Symmetric

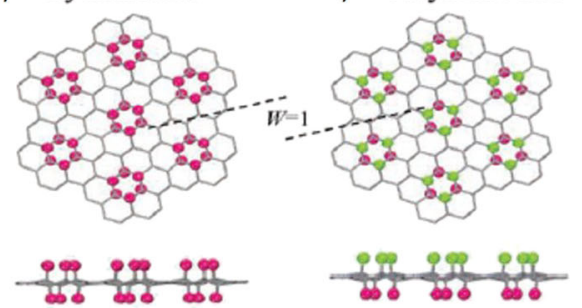

iii) $\mathrm{H}-\mathrm{C}-\mathrm{H}$

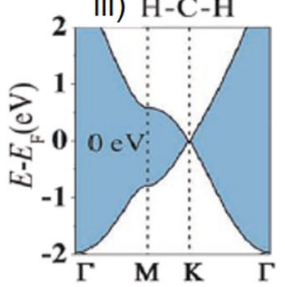

iv) $\mathrm{F}-\mathrm{C}-\mathrm{F}$

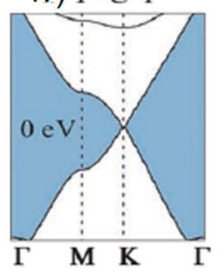

v) $\mathrm{Cl}-\mathrm{C}-\mathrm{Cl}$
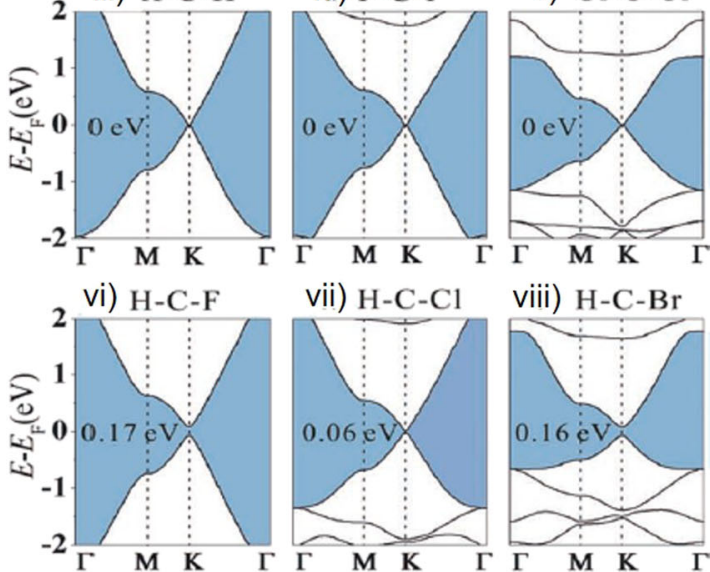

vii) $\mathrm{H}-\mathrm{C}-\mathrm{Cl}$

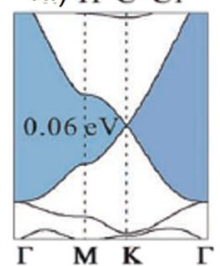

ix) $\mathrm{F}-\mathrm{C}-\mathrm{Cl}$

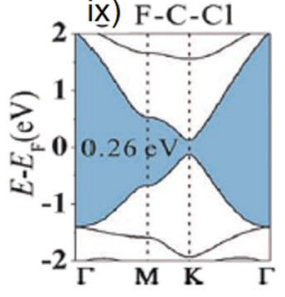

x) $\mathrm{F}-\mathrm{C}-\mathrm{Br}$

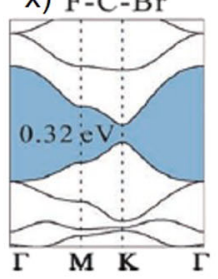

viii) $\mathrm{H}-\mathrm{C}-\mathrm{Br}$

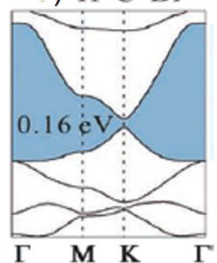

xi) $\mathrm{C} 1-\mathrm{C}-\mathrm{Br}$

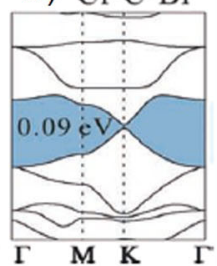

b)

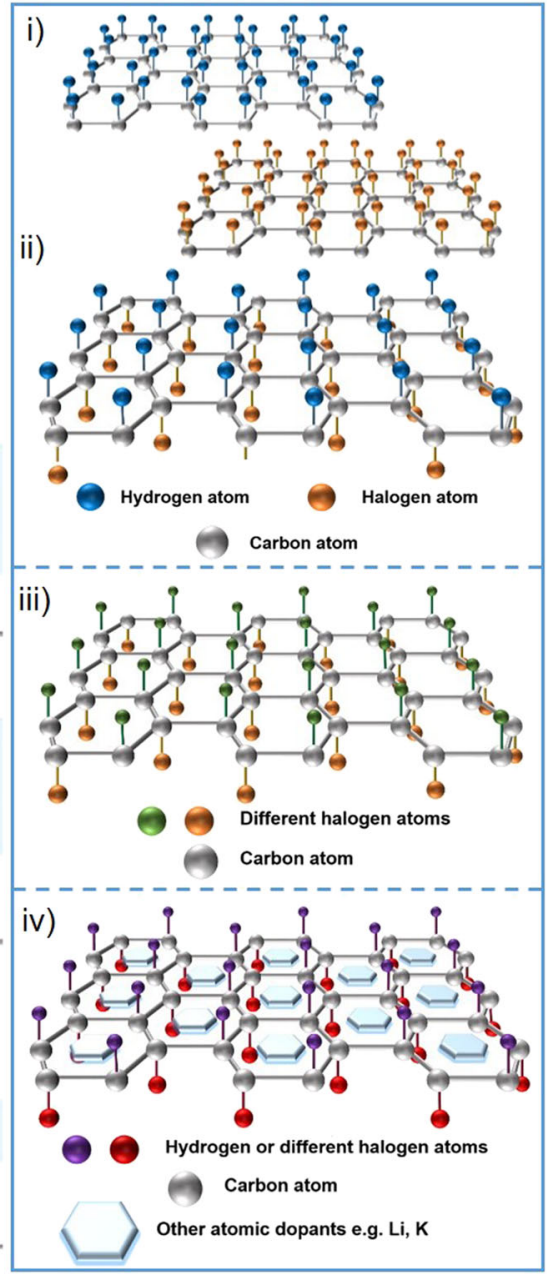

Fig. 1 Janus graphene modified via hydrogenation and halogenation. a Comparisons between the band structures of graphene with symmetric and asymmetric honeycomb pattern modifications with wall width $W=1$. (i, ii) Schematic diagrams of symmetric and asymmetric modifications. (iii-xi) Band structures for various systems, showing the obtained gaps ${ }^{74}$. Adapted from ref. ${ }^{74}$, reproduced with permission (৫) 2013, AIP). b Theoretically favorable Janus graphene structures modified with different combinations of hydrogen, halogen atoms and other atomic dopants on the opposing side, including (i) graphone and single-side halogenated graphene, (ii) hydro-halogenated graphene, (iii) asymmetric halogenated graphene and (iv) hydro-halogenated graphene combined with dopants

In 2010, Robinson et al. ${ }^{84}$ reported the first study of single-side fluorinated graphene by exposing CVD-grown graphene on $\mathrm{Cu}$ foil to a $\mathrm{XeF}_{2}$ atmosphere. They found that the $\mathrm{F}$ coverage saturated at $25 \%\left(\mathrm{C}_{4} \mathrm{~F}\right)$, yielding an optically transparent material over 6 orders of magnitude more resistive than conventional graphene. However, when graphene was placed on a silicon-on-insulator, double-side fluorination of the graphene occurred due to the etching of silicon by $\mathrm{XeF}_{2}$. The resultant perfluorinated graphene demonstrated a structure of two F atoms per one unit cell, forming an empirical structure of perfluorographane (CF). A similar double-sided structure was also reported by Nair et al. ${ }^{85}$ in 2010, in which they exposed graphene supported by an $\mathrm{Au}$ grid to a $\mathrm{XeF}_{2}$ atmosphere. They analyzed the fluorination mechanism in more detail: graphene was exposed to atomic $\mathrm{F}$ formed by the decomposition of $\mathrm{XeF}_{2}$. The $\mathrm{XeF}_{2}$ approach has a clear advantage with respect to possible fluorination in plasma because it avoids potential damage from ion bombardment.

In 2011, Wu et al. ${ }^{86}$ reported the synthesis of singlesided chlorinated graphene. In this work, mechanically exfoliated graphene, CVD graphene and graphene nanoribbons transferred to a $\mathrm{SiO}_{2} / \mathrm{Si}$ substrate were exposed to $\mathrm{Cl}_{2}$ plasma. Importantly, it was found that short periods of $\mathrm{Cl}$ plasma exposure $(<1 \mathrm{~min})$ resulted in the p-doping of graphene without destruction of the basal plan, which led to an increased conductance of the Cl- 

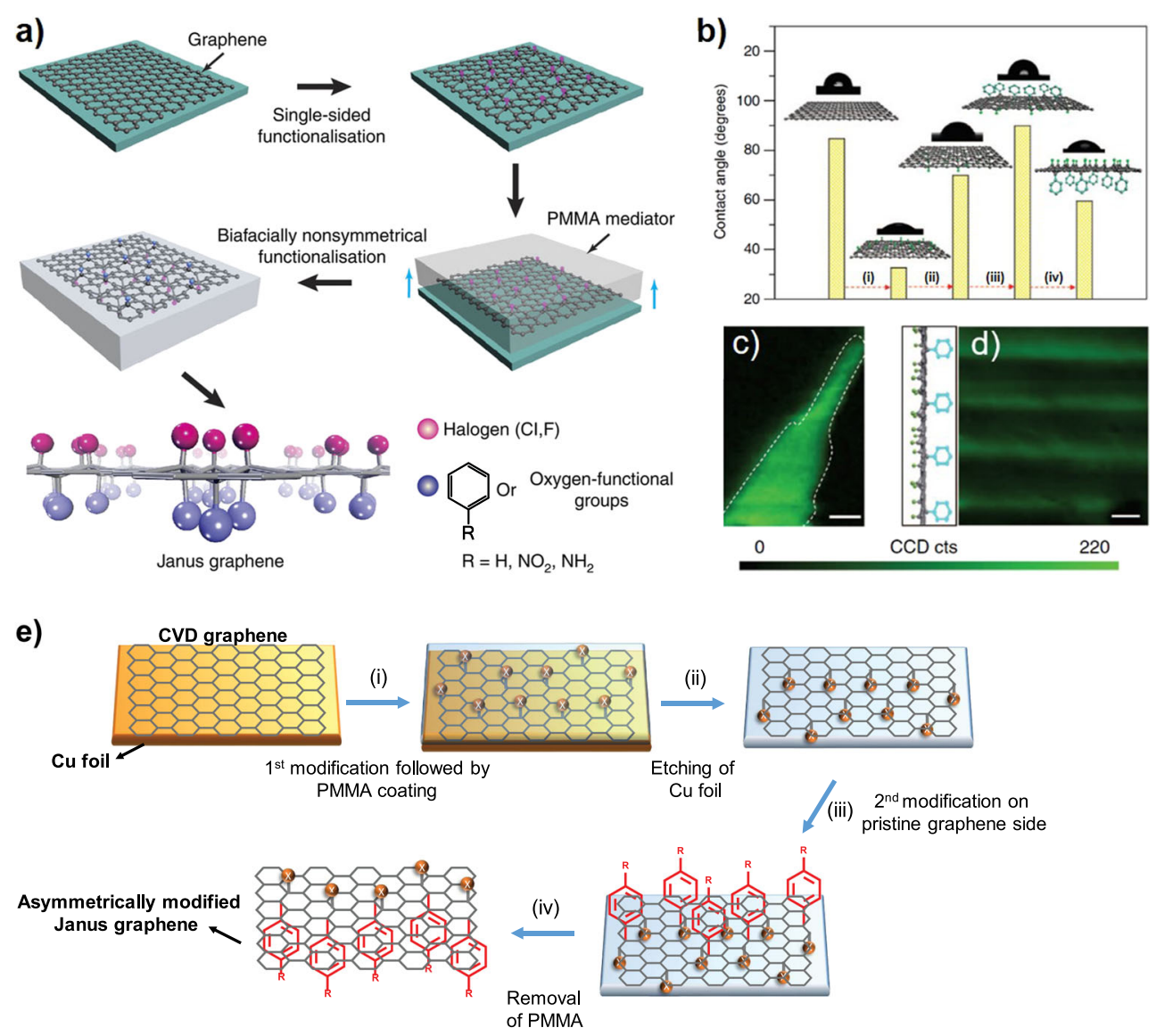

Fig. 2 Synthesis of Janus graphene. a Schematic illustration of the PMMA-assisted transfer procedure to synthesize Janus graphene. $\mathbf{b}$ Variations in the surface wettability of the modified graphene surface dependent on the fabrication procedures. (i) Single-sided photochlorination; (ii) exposure of fresh graphene side; (iii) single-sided phenylation; and (iv) Janus graphene. The insets exhibit images of water droplets and the schematic illustrations of the related surfaces. $\mathbf{c}$ Intensity map of the D band for Janus graphene, demonstrating the uniform distribution of the chemical decorations. The white dotted curve shows the position of the graphene flake. Scale bar, $5 \mu \mathrm{m}$. $\mathbf{d}$ The D-band map for a patterned Janus graphene structure, which is illustrated schematically on the left. Scale bar, 5 mm $^{87}$. Modified after ref. ${ }^{87}$ (๑ 2013, Nature Publishing Group). e Schematic diagram showing common routes for the fabrication and transfer of an asymmetrically modified Janus graphene film

functionalized graphene. Contrary to the effects of $\mathrm{Cl}_{2}$ plasma, the authors also demonstrated that $\mathrm{F}_{2}$ or $\mathrm{H}_{2}$ plasma resulted in the rapid destruction of graphene and loss of conductance.

In 2013, Zhang et al. ${ }^{87}$ reported the synthesis of Janus graphene by photohalogenation. Mechanically exfoliated graphene and CVD graphene supported by a $\mathrm{Si} / \mathrm{SiO}_{2}$ wafer were exposed to a $\mathrm{Cl}_{2} / \mathrm{N}_{2}$ mixture. A xenon lamp $\left(0.9 \mathrm{~W} \mathrm{~cm}^{-2}\right)$ was employed to initiate splitting of chlorine molecules into highly reactive chlorine radicals, which were consequently combined with graphene via a free radical addition reaction (Fig. $2 \mathrm{a}-\mathrm{d})^{87}$. With a poly(methyl methacrylate) (PMMA) layer protecting the halogenated side, the chlorinated graphene could be peeled from the substrate, and the bottom pure carbon side could be exposed for further modification, as shown in Fig. 2e. Additionally, this Janus-functionalized graphene was found to exhibit different wettability properties on the different sides.

\section{Surface modification with small molecules}

To date, covalent modification of graphene with small molecules has been largely limited to the use of diazonium salts. The reaction is due to the spontaneous electron transfer from the graphene layer and its substrate to the diazonium salt. This reduction of diazonium salts has been widely utilized for grafting acryl groups onto the surface of $\mathrm{sp}^{2}$-hybridized carbon materials including glassy carbon ${ }^{88}$, highly ordered pyrolytic graphite $(\mathrm{HOPG})^{89}$, and carbon nanotubes(CNT) ${ }^{90,91}$.

\section{Covalent modification with small molecules}

In 2009, Bekyarova et al. ${ }^{92}$ prepared a Janus graphene layer epitaxially grown on a $\mathrm{SiC}$ substrate by utilizing 


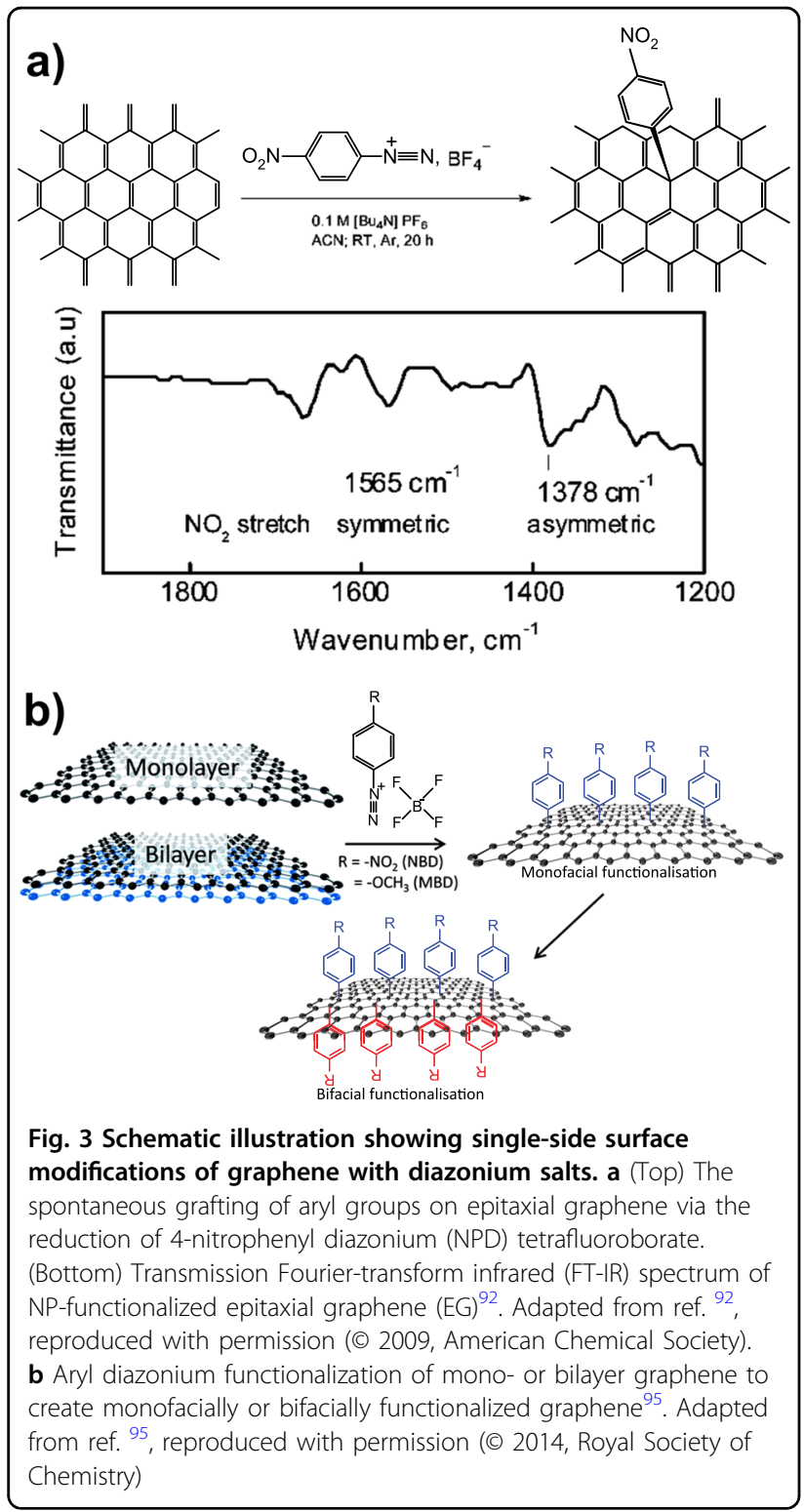

diazonium salts (Fig. 3a). The aryl groups of 4-nitrophenyl diazonium (NPD) tetrafluoroborate attached to graphene were changed from near-metallic to semiconducting. Sinitskii et al. ${ }^{93}$ further noted that the conductivity of monolayer graphene nanoribbons (GNRs) gradually decreased with the diazonium treatment time (4-nitrobenzenediazonium tetrafluoroborate (NBD)), which could be explained by the covalent attachment of 4-nitrophenyl groups to the GNRs, transforming the hybridization of graphene carbon atoms from $\mathrm{sp}^{2}$ to $\mathrm{sp}^{3}$. Moreover, this proved that diazonium functionalization could be a controllable way to tune the electrical properties of graphene. Interestingly, a contradicting result was reported by Farmer et al., ${ }^{94}$ in which graphene devices treated with a 4-bromobenzene diazonium salt did not show any altered conductance at the neutrality point. This suggested that functionalization with different aryl diazonium salts could produce GNRs with differing electrical properties; changing the group in the para position of the phenyl group could modulate the doping-like effects.

In 2013, Zhang et al. ${ }^{87}$ first used such diazonium chemistry to further modify a single-sided chlorinated graphene (as discussed above in the section "Asymmetric functionalization of graphene with halogens and hydrogen") to form dual-functionalized asymmetric graphene sheets. As shown in Fig. 3b, Bissett et al ${ }^{95}$ further applied such diazonium functionalization on both sides of CVD monolayer /bilayer graphene with different aryl diazonium salts in 2014. In this case, graphene films on $\mathrm{SiO}_{2}$ substrates were first exposed to an electron-donating (n-type) dopant 4-methoxybenzenediazonium tetrafluoroborate (MBD) solution for the first reaction on one side. Subsequently, the reacted side was protected by polydimethylsiloxane(PDMS), and the unreacted side was exposed to an electron-withdrawing (p-type) dopant, $\mathrm{NBD}$, for the second functionalization. Interestingly, the net doping of the two-side modified graphene was found to be p-type, indicating that the number of NBD molecules covalently bonded to the graphene was larger than the number of MBDs, thus indicating that modification of one side of the graphene plane could lead to an increase in the reactivity of the opposite side. Moreover, their results showed that dual-faced Janus functionalization induced a significantly higher level of doping than the mono-facial and symmetrically functionalized graphene, allowing for electronic structure control with a larger range for both monolayer and bilayer graphene.

\section{Non-covalent modification with small molecules}

Coletti et al. ${ }^{96}$ demonstrated that the band structure of epitaxial graphene (EG) on $\mathrm{SiC}$ (0001) can be precisely tailored by functionalizing the graphene surface with tetrafluorotetracyanoquinodimethane (F4-TCNQ) molecules in a non-covalent way ${ }^{96}$. Interestingly, from the X-ray photoelectron spectroscopy (XPS) height data, these molecules were considered to stand upright on the surface when densely packed. This is believed to be caused by the removal of electrons from the graphene layer via the cyano groups leading to the formation of a charge-transfer complex between the graphene film and the F4-TCNQ molecular overlayer, allowing for the achievement of charge neutrality in monolayer and bilayer graphene. In bilayer graphene, the hole doping even allowed the Fermi level to shift into the energy bandgap, and the additional dipole developed at the interface with the F4-TCNQ layer caused the bandgap magnitude to increase to more than double its original value. Thus, the 
electronic structure of the graphene bilayer can be precisely tuned by varying the molecular coverage.

\section{Surface grafting with functional polymers Covalent grafting of polymers}

Because of the ample flexibility in molecular design and the high density of the functional groups of polymer chains, grafting functional polymers onto one side or both sides of graphene has become an increasingly popular approach to realize Janus graphene. In particular, taking advantage of the abundant oxygen-containing groups of graphene oxide, researchers have made enormous progress in the covalent grafting of polymers via in situ polymerization reactions.

Self-initiated photografting and photopolymerization (SIPGP) is a facile polymerization method in which styrene, maleic anhydride and several acrylate-type monomers are excited by free radicals under UV radiation ${ }^{97,98}$. These activated monomers initiate a free radical polymerization reaction and create radical sites for surfaceinitiated polymerization on GO by abstracting hydrogen radicals from its surface functional groups such as hydroxyl and epoxy groups ${ }^{99}$. Because an additional initiator is unnecessary and an abundance of monomers are available, SIPGP is a very effective way to functionalize graphene. In asymmetric functionalization, Steenackers et al. ${ }^{100}$ demonstrated single-sided chemical modification of graphene with styrene via SIPGP. The resultant Raman spectroscopic data revealed that photopolymerization occurred and was primarily limited to the existing defect sites on CVD graphene and epitaxial graphene grown on $\mathrm{SiC}$; there was no detectable disruption of the basal plane conjugation of graphene ${ }^{100}$, i.e., the graphene layer was functionalized without degrading its electronic properties. Furthermore, they reported that after tuning the surface reactivity of graphene via electron-beam-induced carbon deposition due to the low bond dissociation energy for hydrogen abstraction from the carbon deposits, a range of vinyl monomers without styrene and methacrylate groups could be locally grafted via SIPGP.

In another approach, Wu et al. ${ }^{101}$ reported the preparation of Janus GO nanosheets via a covalent "grafting to" method. Using the wax-in-water Pickering emulsion process, monolayer GO sheets were coated onto wax microspheres. Surface grafting of an amino-containing polymer such as poly(propylene glycol) bis(2-aminopropyl ether) was then carried out on the exposed side of GO. After dissolving the wax core with chloroform, singleside-modified GO sheets were released and collected.

\section{Non-covalent grafting of polymers}

Non-covalent attachment of an external substance is another strategy for graphene surface modification, which can functionalize the graphene film while leaving its natural conjugated structure unaffected. This kind of modification of graphene mainly involves the grafting of polymers utilizing van der Waal's forces, hydrogen bonding, coordination bonding and $\pi-\pi$ stacking of aromatic molecules on the graphene plane ${ }^{63}$. Because of the excellent mechanical strength and the hexagonal structure of carbon atoms, pristine monolayer graphene can act as a mechanical support platform for functional polymer brushes through $\pi-\pi$ interactions. In 2009, to achieve a suitable surface wettability of CVD graphene for uniform conductive polymer coating while maintaining the conductivity for use as an electrode in an organic solar cell, Wang et al. ${ }^{7}$ modified CVD graphene with selfassembled pyrenebutanoic acid succinimidyl ester, which was able to stack on the graphene surface in a face-to-face orientation via the pyrene group ${ }^{7}$.

The grafting of polymer brushes onto CVD graphene films in a similar non-covalent manner was then demonstrated by Xiao et al. ${ }^{102}$ in 2013 . Through microcontact printing, a patterned $\mathrm{NH}_{2}$-terminated pyrene derivative layer $\left(\mathrm{Py}-\mathrm{CH}_{2} \mathrm{NH}_{2}\right)$ was transferred onto a Sisubstrate-supported graphene layer and successfully anchored to the surface via $\pi-\pi$ stacking interactions between pyrene groups and the graphene scaffold. With the amino groups pointing towards the outer face, photopolymerization could be carried out. By SIPGP, polystyrene (PS) brushes $(\sim 200 \mathrm{~nm})$ were successfully grafted from the patterned active area, while unpatterned areas on graphene displayed a thinner layer of homogenous PS brushes grafted from existing defect sites.

In 2014, Gao et al. ${ }^{103}$ further demonstrated the singleside decoration of CVD graphene film with polymer brushes by adopting the specific atom transfer radical polymerization (ATRP) macro-initiator poly[2-(2-bromoisobutyryloxy) ethyl methacrylate-co-4-(1-pyrenyl) butyl methacrylate] (Fig. 4a). Such an initiator was found to be anchored onto the graphene because of the strong interaction between the graphene basal plane and the pyrene side chains of the initiator, as indicated in previous studies ${ }^{104,105}$. In this experiment, various polymer brushes were grafted to the initiator-soaked CVD graphene layer grown on $\mathrm{Cu}$ foil. Moreover, it was confirmed that the polymer brush layer was densely grafted while also protecting graphene during the wet etching and transfer process; different kinds of brushes provided specific applications to the modified graphene film, such as smart control of surface wettability and immobilization of biomolecules. This approach thus introduced a robust route for fabricating lightweight, transferrable and functiontailorable 2D organic materials. Similarly, pre-synthesized pyrene-containing polymers can also be directly immobilized on graphene's basal plane through $\pi-\pi$ interactions. In 2016, Yang et al. ${ }^{106}$ adopted PS microspheres as a template for a GO assembly through electrostatic 


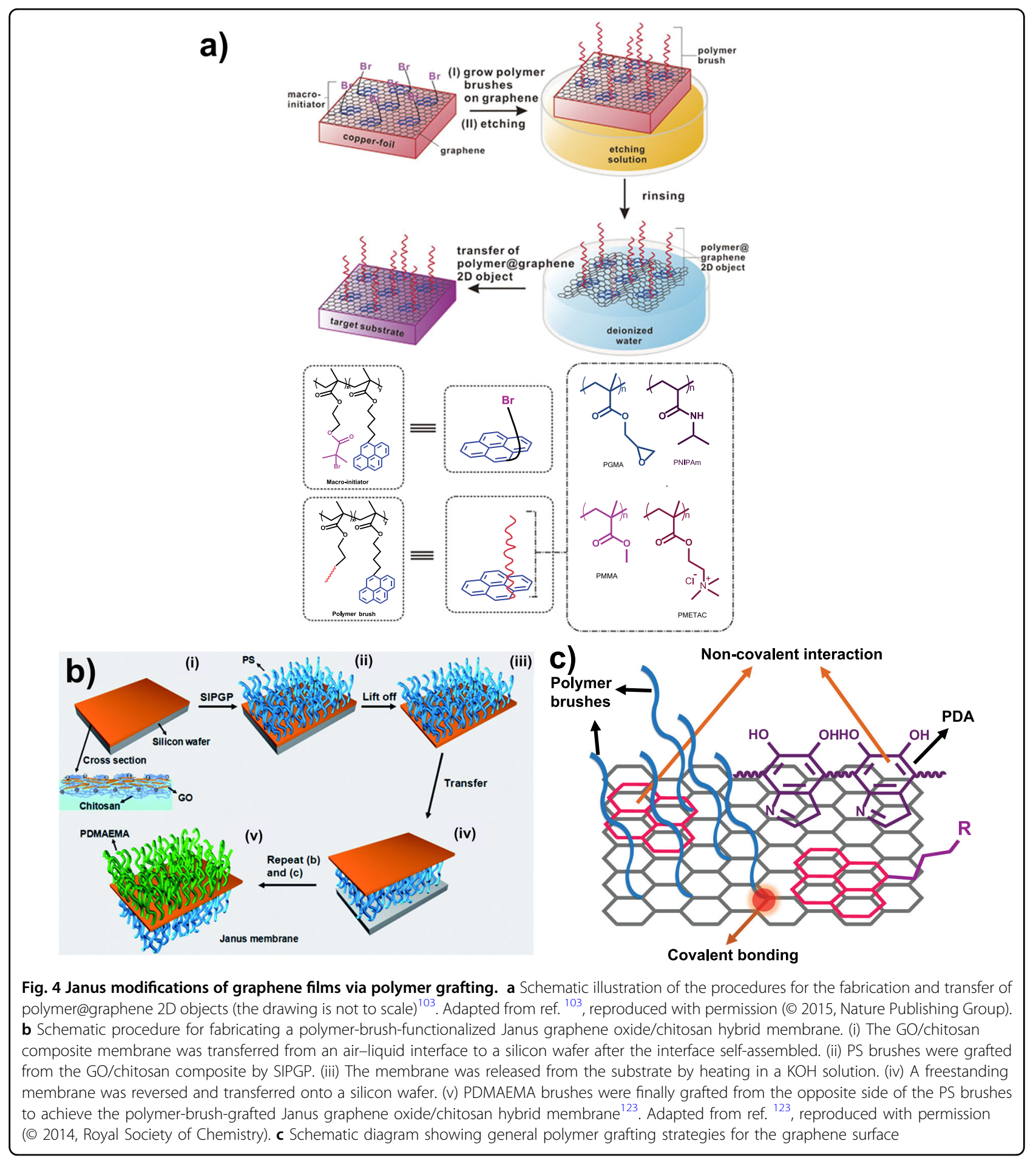

interactions, and pyrene-terminated poly(2-(dimethylamino)ethyl methacrylate) (PDMAEMA) chains were attached to the exposed side of GO. After dissolving the PS core, free PS chains were attached to the inner side of the GO spheres, giving amphiphilic Janus GO nanosheets in the form of PS-GO-PDMAEMA that can be used as a surfactant for stabilizing oil droplets dispersed in water ${ }^{106}$.
Apart from pyrene groups, polydopamine has also been widely utilized for the facile and damage-free surface treatment of low-surface-energy materials, such as pristine graphene, as shown in Fig. 4c, because of the nonspecific adherence of dopamine to a wide variety of surfaces. This spontaneous adsorption of polydopamine caused a significant decrease in the water contact angle of 
graphene ${ }^{107}$. Thus, polymers that do not normally wet pristine graphene could be readily deposited after surface modification with dopamine. For example, Kim et al. demonstrated the formation of a thin layer of neutral poly(styrene-ran-methylmethacrylate) (P(S-r-MMA)) on polydopamine-modified CVD graphene through spin coating, followed by a thermal annealing step to covalently react the hydroxyl groups of the copolymer with the catechol groups of the polydopamine. In another example, Park et al. ${ }^{108}$ further directed the self-assembly of polystyrene-block-polymethylmethacrylate (PS- $b$ PMMA) on polydopamine-modified graphene. In their experiment, a neutral layer of $\mathrm{P}(\mathrm{S}-r$-MMA) was first deposited on graphene, as discussed in ref. ${ }^{104}$. PS- $b$ PMMA was then self-assembled onto the neutralized layer to form vertical lamellar or hexagonal cylinder-like nanostructures based on the PS/PMMA ratio. Such nanostructured thin films were used as a resist for patterning graphene through reactive ion etching to fabricate graphene nanoribbons and nanomeshes, which could be applied as the channels of field-effect transistors (FETs).

\section{Deposition with metals and metal oxides Vacuum deposition of ultrathin films}

Apart from the organic moieties that provide ample surface functionality of Janus graphene, metals and metal oxides can render graphene with specific electrical and chemical properties that even organic moieties cannot offer. For the formation of Janus graphene with an ultrathin layer $(<10 \mathrm{~nm})$ of metal/metal oxides, physical vapor deposition is always the most straightforward approach. A series of metals including $\mathrm{Au}, \mathrm{Ag}$ and $\mathrm{Cu}$ have been deposited on CVD graphene effectively via different vacuum deposition techniques ${ }^{109}$. The film structures were largely dependent on the deposition conditions, film thickness, topography and the number of graphene layers. For example, it was found that the grain size of $\mathrm{Au} \quad(<5$-nm-thick film made via thermal deposition) was positively dependent on the number of graphene layers, while the grain density decreased with increasing graphene layers ${ }^{110}$. With sputtering, the particle size $(<2 \mathrm{~nm})$ could be precisely controlled by adjusting the operation angle, current and deposition time ${ }^{111}$.

Atomic layer deposition (ALD) is a powerful tool for depositing uniform and ultrathin $(\AA)$ metal or metal oxide layers with precise thickness control because of its selflimiting surface reaction nature ${ }^{112,113}$. However, additional chemical treatment is needed for ALD to occur on pristine graphene because of its inertia with respect to the nucleation of inorganic particles in the ALD process. For example, the nucleation of $\mathrm{Al}_{2} \mathrm{O}_{3}$ on graphene's basal plane has been facilitated by a gaseous phase pretreatment of pristine graphene with 3,4,9,10-perylene tetracarboxylic acid ${ }^{113}, \mathrm{NO}_{2}{ }^{114}$ or ozone ${ }^{114}$, which functionalized the graphene surface through the introduction of a multitude of non-destructive, reactive surface groups.

\section{Solution deposition of nanoparticles}

Different from thin films, nanoparticle-decorated Janus graphene is typically synthesized via solutionbased chemical approaches. Taking advantage of the presence of oxygen-containing groups on chemically exfoliated and modified graphene sheets, metal oxide nanoparticles (NPs), e.g., ZnO NPs, could easily anchor onto the graphene surface by simply immersing the graphene film in the NP solution ${ }^{115}$. Furthermore, with a suitable surface functionalization, this method could also be extended to metal NPs such as Au NPs (Fig. 5) ${ }^{115}$.

In contrast, to decorate graphene-based films with metal NPs, electroless deposition was demonstrated as an efficient and convenient way. As reported by Lu et al. ${ }^{116}$ in 2011, an rGO layer coated on a Si wafer was successfully decorated with $\mathrm{Ag}$ or Au NPs by a simple immersion step in a mixture of $\mathrm{HF}$ and $\mathrm{AgNO}_{3}$ or $\mathrm{HAuCl}_{4}$. As the exposed $\mathrm{Si}$ parts were slightly etched by the hydrofluoric acid, they donated electrons that triggered the reduction of positive metal ions to form the corresponding metal NPs. Later, Yin et al. ${ }^{117}$ reported the deposition of Pt NPs on an rGO layer by photochemical reduction ${ }^{117}$. In this case, an rGO-coated $\mathrm{Si} / \mathrm{SiO}_{2}$ wafer was immersed in a solution containing $\mathrm{PtCl}_{4}{ }^{2-}$ and ethanol under light irradiation. Because of the oxidation of alcohols and the difference in reduction potentials between $\mathrm{PtCl}_{4}{ }^{2-}(+0.775 \mathrm{~V}$ vs. standard hydrogen electrode (SHE)) and rGO (+0.38 V vs. SHE), chloro-platinum complexes captured electrons from the solution, as well as the rGO surface, and were reduced to $\mathrm{Pt}^{0}$.

In 2015, an in situ galvanic displacement method for the asymmetric decoration of CVD graphene with noble metal (Au, Pt, Pd)NPs was reported by Toth et al. ${ }^{118}$. In their work, CVD graphene supported by $\mathrm{Cu}$ foil was immersed into the noble metal precursor solution. Galvanic displacement of the metallic NPs ( $\mathrm{Au}, \mathrm{Pt}$ and $\mathrm{Pd})$ occurred on the upper surface of CVD graphene, where the $\mathrm{Cu}$ foil acted as an electron donor that transferred electrons to the precursor salts $\left(\mathrm{AuCl}_{4}{ }^{-}, \mathrm{PdCl}_{4}{ }^{2-}\right.$ and $\left.\mathrm{PtCl}_{4}{ }^{2-}\right)^{119}$. To further deposit additional metallic NPs on the bottom side, the $\mathrm{Cu}$ foil was etched away and the decorated upper side of graphene was floated at the interface of an organic reducing agent and an aqueous solution containing the desired precursor salt of the second metal. Thus, the second deposition occurred at the aqueous-organic liquid/liquid interface, yielding monolayer graphene bifacially deposited with different noble metal nanoparticles. 


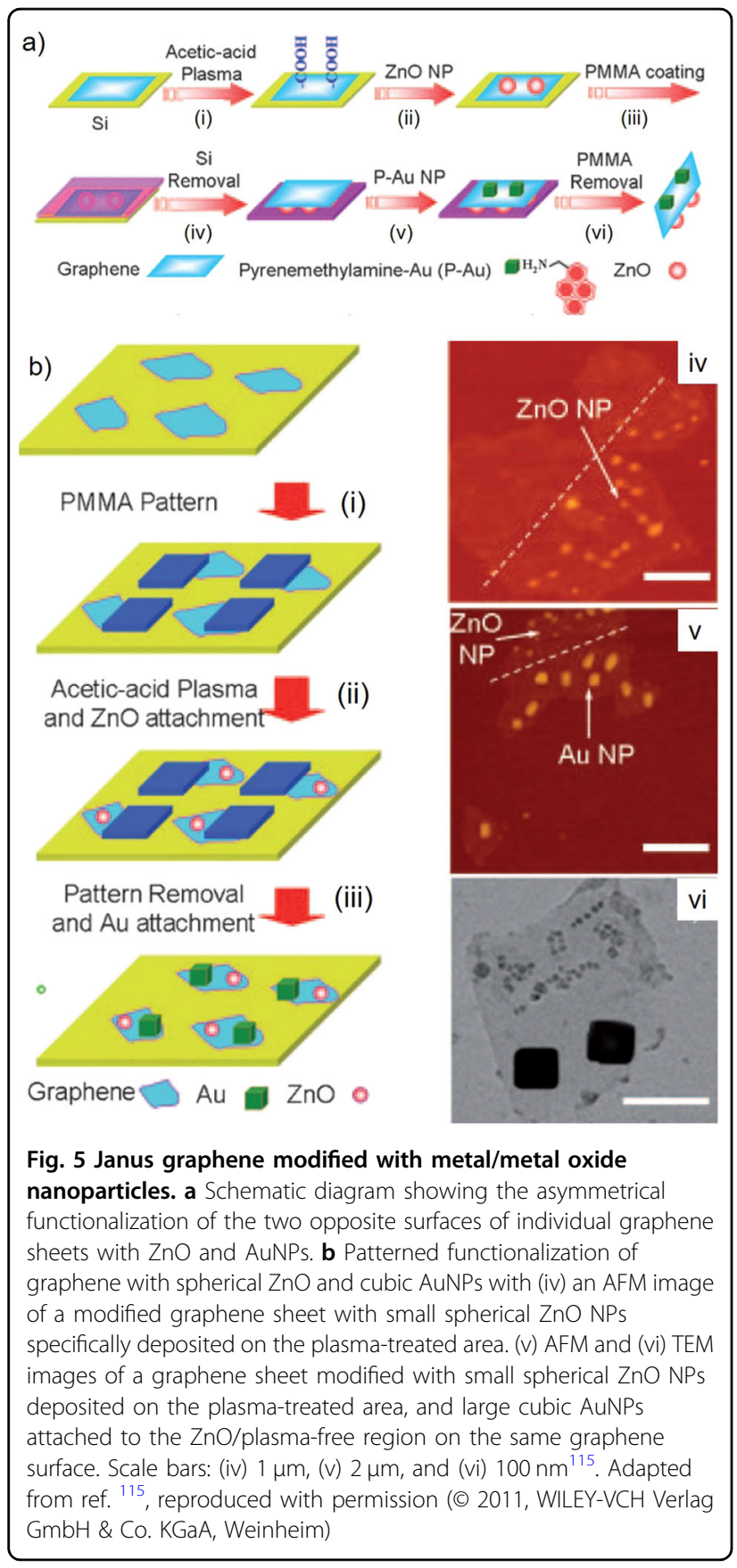

\section{Preparation of Janus graphene-based thin films}

Janus graphene-based thin films are the other major type of graphene-based Janus materials. In this category, thin films made of solution-exfoliated graphene nanosheets, GO, rGO and their composites, are fabricated first, followed by asymmetric surface modification to yield the Janus thin films. In the literature, there have been many review articles on how to prepare graphene-based thin films ${ }^{120,121}$. In this section, we only focus on how to asymmetrically modify the pre-made thin films into Janus thin films.

In principle, the synthetic routes discussed in the section "Synthesis of Janus graphene" are all applicable surface modification techniques for the materials mentioned in this section. Nevertheless, it should be noted that most graphene-based thin films are made of $\mathrm{GO}$ and $\mathrm{rGO}$ (mainly because of their superb solution processability), which are rich in oxygen-containing groups. Therefore, most works reported in this section rely on these functional groups for surface reactions. Several representative examples are discussed below.

In 2014, Xiao et al. ${ }^{122}$ developed a robust strategy to fabricate micro-patterned GO-based polymer brushes with adjustable sizes and shapes over a large area. Based on the strong supramolecular interaction from the formation of multiple hydrogen bonds between GO nanosheets and the HO-terminated surface, GO nanosheets were printed from a PDMS stamp onto a Si substrate. The photoactive site on the GO surface allows for the growth of polymer brushes with well-designed patterns by SIPGP. To prove the concept, PS brushes were grafted onto micropatterned GO films, and a nearly defect-free PS brush layer was observed with a well-controlled height $(\sim 60 \mathrm{~nm})$ and a feature spacing of $10 \mu \mathrm{m}$ over a large area. By immersing the PS-modified substrate in an aqueous $\mathrm{KOH}$ solution, the polymer brush grafted GO microfilms were released from the silicon surface and became readily available for transfer. On the other hand, as illustrated in Fig. 4b, Han et al. ${ }^{123}$ demonstrated the production of Janus graphene/chitosan hybrid sheets by asymmetrically grafting two sides of the hybrid sheets with PS and PDMAEMA brushes. GO nanosheets with negative charges behave like negatively charged polyelectrolytes that can interact with positively charged polyelectrolytes to form a stable complex. In this work, the $\mathrm{GO} /$ chitosan composite membrane was prepared through the interfacial self-assembly of $\mathrm{GO}$ with the positively charged chitosan solution. Using SIPGP, one side of the composite membranes was first grafted with PS, and then the other side of the composite was grafted with PDMAEMA. Because of the large difference in surface wettability of these two polymers, a membrane with contrasting hydrophilicity on the top and bottom sides was fabricated. In addition, a patterned carbon-based hybrid Janus thin film was demonstrated by Xiao et al. $^{6}$ using a microcontact printed CNT/GO film on a Si wafer as the starting material. PDMAEMA brushes were grafted on the upper side of the hybrid films via SIPGP. After etching the natural $\mathrm{SiO}_{2}$ surface layer of the substrate, the freestanding hybrid films were released from the substrate and could be transferred to any other target substrate. In this way, the polymer-grafted side acted as an insulating carpet, while the carbon side acted as a conductive 
element, forming a flexible and miniature electric carpet with potential application as a localized heating element in micro-/nano-devices.

Electrochemical deposition is another effective and facile approach for surface modification. Since most graphene-based thin films are highly conductive, electrochemical deposition can be carried out directly on the surface to achieve asymmetric modification in one step. In 2010, Wu et al. ${ }^{124}$ demonstrated the deposition of a series of metal oxides, including $\mathrm{ZnO}$ nanorods, as well as p-type and n-type $\mathrm{Cu}_{2} \mathrm{O}$ films, onto $\mathrm{rGO}$ thin films that were supported on a polyethylene terephthalate (PET) substrate. For these kinds of Janus thin films, the conductive rGO was used as a transparent electrode, while the semiconducting metal oxide was used as the active absorber of the photovoltaic device.

The $\mathrm{pH}$-responsive Janus platelets with the two faces orthogonally functionalized with PS and poly(acrylic acid) (PAA) were demonstrated by McGrail et al. ${ }^{125}$. Acrylate functionalities were first appended to both faces of GO using acrylonitrile in an acidic aqueous environment to give Acr-GO-Acr. These platelet products would lie at the interface between oil and water phases, so they were introduced to a toluene-water interface with watersoluble PAA5-SH and oil-soluble PS40-SH. The double thiolene reaction yielded PS40-GO-PAA5 Janus sheets, which were useful in stabilizing emulsions at high $\mathrm{pH}$. However, under acidic conditions ( $\mathrm{pH} \sim 2$ ), PS40GO-PAA5 does not stabilize emulsions because of the protonation of carboxylic acid moieties decreasing the solubility of PAA in water.

\section{Applications of graphene-based Janus materials Surface functionalization}

Because of the two-dimensional structure of graphenebased Janus materials, one major application is to transfer these functional 2D objects onto target substrates for the modification of surface wettability and functionality.

Both Janus graphene and Janus graphene-based thin films have been reported for this purpose. In general, these 2D objects are lifted off the original substrate into a liquid bath and then fished onto a target substrate. It should be noted that if the 2D objects are too thin, e.g., a few nanometers, a PMMA sacrificial layer is normally needed to assist the transfer process. For example, Zhang et $a{ }^{87}$ synthesized a Janus graphene, of which one side was modified by chlorination and the other side was modified by phenylation. With a PMMA-assisted transfer, the Janus graphene exhibited bifacially anisotropic wettability: the contact angle was $89.9^{\circ}$ on the phenylated side and $59.6^{\circ}$ on the chlorinated side. In another example demonstrated by Wang et al. ${ }^{126}$, fluorinated graphene transferred to $\mathrm{SiO}_{2} / \mathrm{Si}$ and quartz substrates was achieved after PMMA removal and exposure to $\mathrm{XeF}_{2}$ in an inert atmosphere. Such fluorinated graphene was shown to enhance the cell adhesion, growth and proliferation of mesenchymal stem cells (MSCs) and to generate a neuroinductive effect via spontaneous cell polarization. Through the rapid and large-area printing of PDMS ink, cell neurogenesis could also be enhanced via channelconfined cell elongation without any chemical factor stimulation (Fig. 6).

In another work, Gao et al. ${ }^{103}$ synthesized polymerbrush-grafted CVD graphene, namely, polymer@graphene 2D objects, through surface-initiated ATRP on a CVD graphene surface. From the abundant possibility of monomers, they were able to demonstrate a wide variety of polymer@graphene 2D objects with tunable polymer thicknesses and functionality. With thermal responsive poly ( $N$-isopropylacrylamide) (PNIPAm), the transferrable 2D objects typically showed a lower critical solution temperature (LCST) behavior: the contact angle was $\sim 60^{\circ}$ when the temperature was lower than the LCST of PNIPAm and $\sim 80^{\circ}$ when the temperature was higher than the LCST. In addition, they prepared poly(glycidyl methacrylate) on graphene; this $2 \mathrm{D}$ object was used as a reactive and transparent platform for the immobilization of biomolecules such as DNA and proteins for the fabrication of bio-microarrays.

Moreover, by adopting a Ag/AuNP-decorated rGO layer on a $\mathrm{Si}$ substrate, as mentioned in the section "Solution deposition of nanoparticles", a surfaceenhanced Raman scattering substrate was developed by $\mathrm{Lu}$ et al. ${ }^{116}$. The $\mathrm{Ag} / \mathrm{AuNP}$-coated $\mathrm{rGO}$ layer was proven to exhibit very strong adsorptions of aromatic molecules (e.g., rhodamine 6G, methyl violet, rhodamine $\mathrm{B}$ and methylene blue), enhancing their Raman signal and allowing for the detection of aromatic molecules with low detection limits (nM level). On the other hand, a highly efficient surface plasmon resonance (SPR) immunosensor was developed by Singh et al. ${ }^{127}$ with Janus graphene transferred to $\mathrm{Au}$. In their experiment, coppercoordinated nitrilotriacetic acid containing terminal pyrene groups was used to functionalize monolayer CVD graphene, and then the Janus graphene was transferred onto an $\mathrm{Au}$ substrate. The aim of this approach was to amplify the SPR signal and to control the immobilization of a biotinylated cholera toxin antigen. The results showed that the addition of a single Janus graphene sheet increased the SPR sensor performance by $80 \%$ compared with the graphene-devoid setup.

\section{Field-effect transistors (FETs)}

As mentioned in previous sections, pure graphene is a zero-bandgap semimetal, which is very difficult to use in FETs because of the high leakage currents and power dissipation ${ }^{128}$. Although covalent modification of graphene can effectively widen graphene's bandgap, it also 
(a)

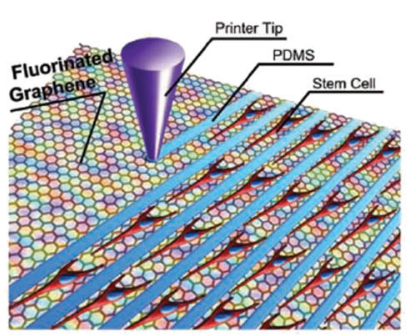

(b)

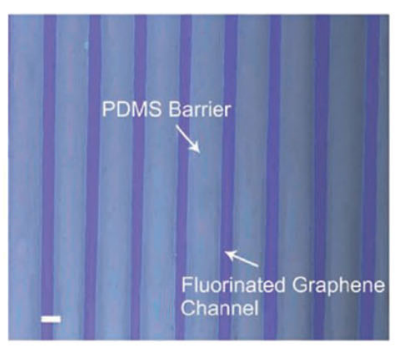

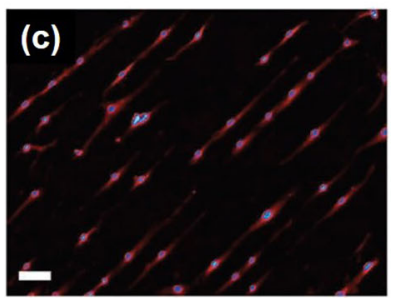

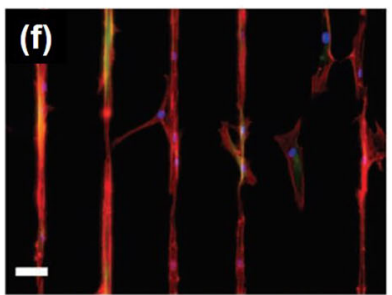

Tuj1 Actin DAPI
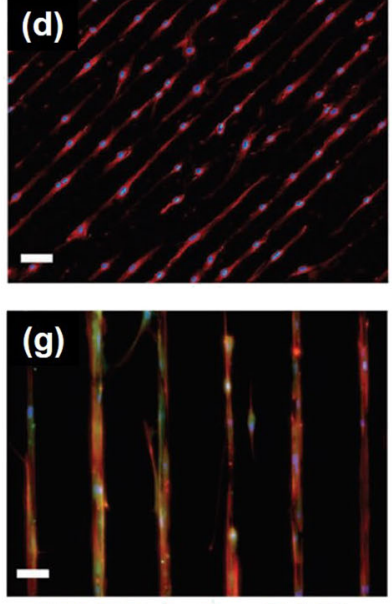

MAP2 Actin DAPI

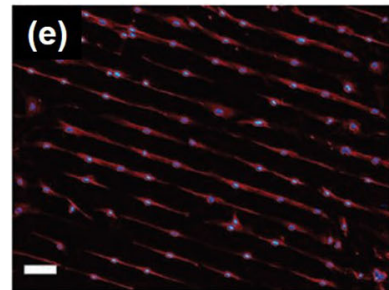

(h)

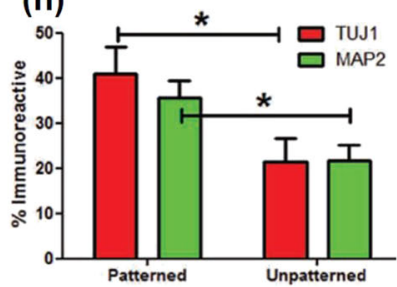

Fig. 6 Patterning on fluorinated graphene film via large-area ink-jet printing method. a Schematic drawing of patterning MSCs by directly printing PDMS barriers on graphene films. b Optical microscope image of printed PDMS on a fluorinated graphene film (scale bar $=50 \mu \mathrm{m})$. $\mathbf{c}-\mathbf{e}$ The aligned growth of stem cells on graphene, partially fluorinated graphene and fluorinated graphene with printed PDMS patterns (scale bar $=100 \mu \mathrm{m}$ ). f, $\mathbf{g}$ MSCs preferentially attached on the FG strips, with their F-actin aligned (red) and expressing neural specific markers-Tuj1 and MAP2 (green) (scale bar $=50 \mu \mathrm{m}$ ). $\mathbf{h}$ The percentage of immunoreactive cells for Tuj1 and MAP2 on unpatterned and patterned FG strips. Note that the patterned FG strips induce higher expression of Tuj1 and MAP2 in the absence of retinoic acid. $(n=6, p<0.05)^{126}$. Adapted from ref. ${ }^{126}$, reproduced with permission (๑ 2012, WILEY-VCH Verlag GmbH \& Co. KGaA, Weinheim)

significantly destroys the basal plane of graphene, which leads to a loss of electronic conductivity and mobility. To avoid this, Sarkar et al. ${ }^{129}$ reported the modification of one side of graphene via a mono-hexahapto $\left(\eta^{6}\right)$-chromium complexation, as shown in Fig. 7. The $\eta^{6}$-mode of bonding is distinct from the modification induced by the conventional covalent $\sigma$-bond formation that involves the creation of $\mathrm{sp}^{3}$ carbon centers in the graphene lattice. This functionalization preserves the conjugation of the extended periodic $\pi$-electron systems, and the functionalized carbon atoms remain part of the electronic band structure, retaining the high mobility of graphene. Thus, FETs made with this Janus graphene retained a high degree of the mobility and showed an enhanced on/off ratio of 5 to $13^{129}$.

On the other hand, protein-functionalized Janus graphene prepared through a non-covalent chemistry could be used for the fabrication of highly sensitive FET biosensors. In 2011, Sudibya et al. ${ }^{130}$ demonstrated a FET sensor of metal ions in aqueous solutions by adopting protein-functionalized rGO films as the active channel. In their experiments, proteins were attached to the micropatterned rGO films via a linking layer, 1-pyrenebutanoic acid succinimidyl ester, which strongly attached its pyrene tail to rGO via $\pi-\pi$ stacking while leaving another end for further covalent bonding with protein molecules. To achieve the specific detection of different kinds of metals, two types of proteins were adopted, namely, calmodulin (CaM) and metallothionein type II protein (MT-II). For CaM-modified rGO-FETs, the field-effect modulation of the rGO-FETs was attributed to the binding of $\mathrm{Mg}^{2+}$ and $\mathrm{Ca}^{2+}$ ions, inducing significant changes in the conductance. The detection limit of $\mathrm{Mg}^{2+}$ and $\mathrm{Ca}^{2+}$ ions was found to be $\sim 1 \mu \mathrm{M}$ with a signal-to-noise ratio of $\sim 20$ to 30 . For the MT-IImodified rGO-FET, the conductance change was introduced by the conformational change of MT when binding with heavy metal ions. This rGO-FET could detect xenobiotic heavy metal ions $\left(\mathrm{Cd}^{2+}\right.$ and $\left.\mathrm{Hg}^{2+}\right)$ with an even lower detection limit of $\sim 1 \mathrm{nM}$ (with a signal-tonoise ratio of $\sim 25$ to 30 for $\mathrm{Hg}^{2+}$ ions and $\sim 15$ to 20 for $\mathrm{Cd}^{2+}$ ions). Compared with the previously reported 

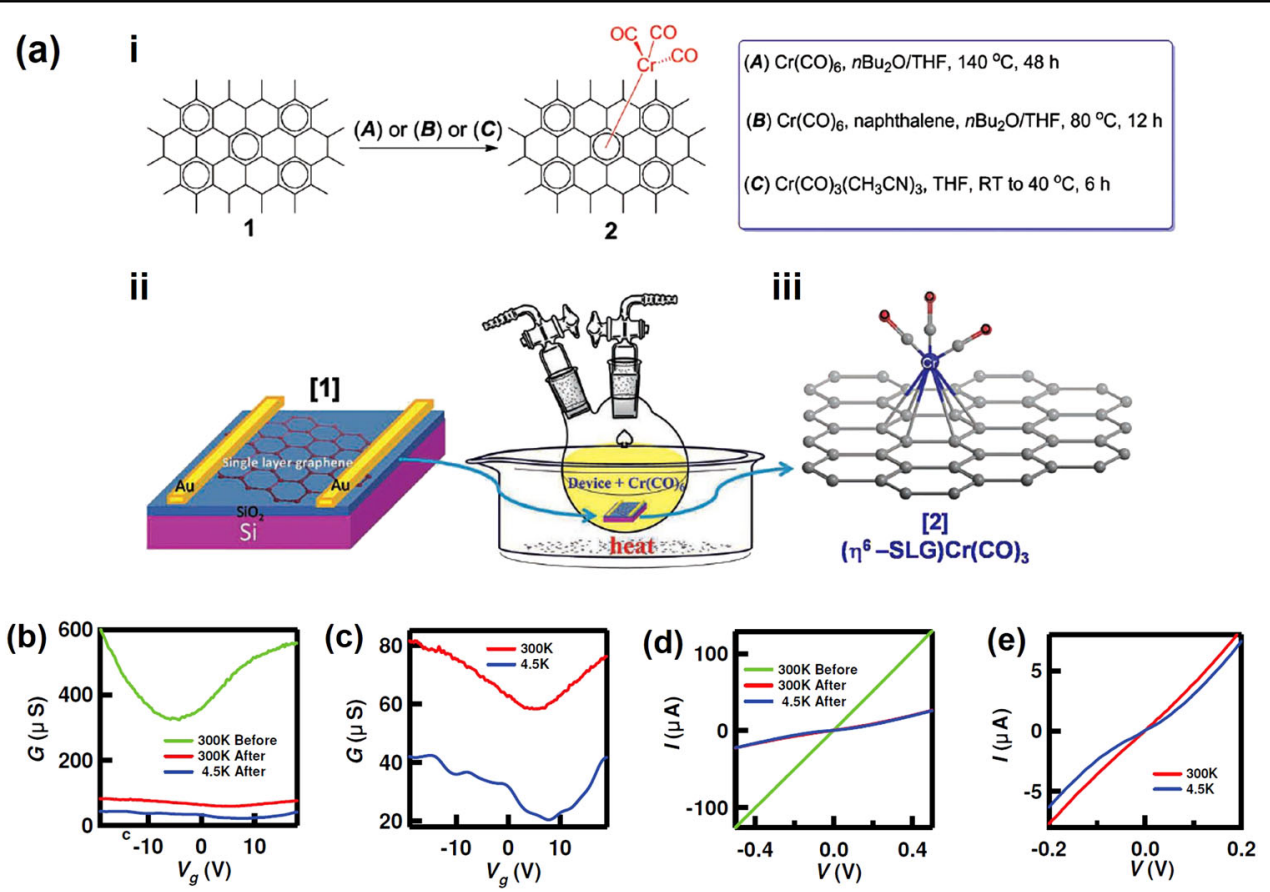

Fig. 7 Single-sided modification of graphene via mono-hexahapto (h6)-chromium complexation. a Organometallic functionalization of singlelayer graphene devices (SLG, 1): (i) schematics of functionalization approaches using three different reaction routes to obtain a hexahapto-chromium complex, $\left(\eta^{6}-\mathrm{SLG}\right) \mathrm{Cr}(\mathrm{CO})_{3}(2)$; routes - $\mathrm{A}: \mathrm{Cr}(\mathrm{CO})_{6}, \mathrm{n}-\mathrm{Bu}_{2} \mathrm{O} / \mathrm{THF}, 140^{\circ} \mathrm{C}, 48 \mathrm{~h}$, under argon; $\mathrm{B}$ : $\mathrm{Cr}(\mathrm{CO})_{6}$, naphthalene, $\mathrm{n}-\mathrm{Bu}{ }_{2} \mathrm{O} / \mathrm{THF}, 80^{\circ} \mathrm{C}, 12 \mathrm{~h}, \mathrm{under}$ argon; and $\mathrm{C}: \mathrm{Cr}(\mathrm{CO})_{3}\left(\mathrm{CH}_{3} \mathrm{CN}\right)_{3}, \mathrm{THF}$, room temperature to $40^{\circ} \mathrm{C}, 6 \mathrm{~h}$, under argon; (ii) illustration of the graphene device and the functionalization process; and (iii) three-dimensional model of the $\left(\eta^{6}-\mathrm{SLG}\right) \mathrm{Cr}(\mathrm{CO})_{3}$ organometallic complex. $\mathbf{b} \mathrm{G}\left(\mathrm{V}_{\mathrm{g}}\right)$ characteristic from a device before and after functionalization. $\mathbf{c} G\left(V_{\mathrm{g}}\right)$ characteristic of the functionalized device at $300 \mathrm{~K}$ and $4.5 \mathrm{~K}$. d I $(\mathrm{V})$ curves of the device before and after functionalization. e I $(V)$ curves of the functionalized device at $300 \mathrm{~K}$ and $4.5 \mathrm{~K}^{129}$. Adapted from ref. ${ }^{129}$, reproduced with permission $(\odot) 2013, \mathrm{WILEY}-\mathrm{VCH}$ Verlag GmbH \& Co. KGaA, Weinheim)

fluorescence-based methods, this rGO-FET sensor provided faster real-time electronic detection of heavy metal ions with higher sensitivity.

In another work reported by Yang et al., ${ }^{131}$ one side of a graphene nanomesh was immobilized with pyrene modified, a HER2-specific aptamer, via the $\pi-\pi$ interaction between pyrene and graphene's basal plane, as shown in Fig. 8. The interactions between the aptamer and HER2 protein can affect the charge carrier density on the surface of the graphene nanomesh (GNM) and allow for label-free detection using a FET configuration. With this Janus graphene, FETs for the selective detection of HER2, a transmembrane protein that is overexpressed by 100 -fold or more on the surface of breast cancer cells, was constructed with a detection limit down to $0.6 \times 10^{-15} \mathrm{M}$. The real-time detection of breast cancer cells using this Janus graphene was also demonstrated.

By using an rGO film as an electrode, monocrystalline $\mathrm{ZnO}$ NRs with a high donor concentration were successfully deposited by an electrochemical method ${ }^{132}$. As a proof-of-concept application, the $\mathrm{rGO} / \mathrm{ZnO} \mathrm{NR}$ assembly was used to fabricate inorganic-organic hybrid solar cells with a layered structure of quartz/rGO/ZnO NRs/P3HT/
PEDOT:PSS/Au. The observed power conversion efficiency(PCE) was $\sim 0.31 \%$, which is higher than that of previously reported solar cells using graphene as an electrode.

\section{Actuators}

Through the asymmetric surface engineering of graphene-based sheets, each side of a 2D Janus graphene sheet can behave very differently under the same external stimuli. Thus, one can trigger the shape change of the $2 \mathrm{D}$ sheets by carefully designing the stress/strain response on each side. To date, most Janus-graphene-based actuators have been designed based on the contrasting hydrophilicity/hydrophobicity given to the opposing sides of the 2D objects.

For example, Xie et al. ${ }^{133}$ developed a Janus-graphenebased electrochemical actuator via direct plasma treatments, as shown in Fig. 9. A large difference in hydrophilicity was created between the two sides of a filtrated rGO film (thickness $\sim 4-5 \mu \mathrm{m}$ ) by exposing one side of the film to a hexane plasma and the opposite side to an $\mathrm{O}_{2}$ plasma treatment. The hexane-plasma-treated side became relatively hydrophobic, which limited the 


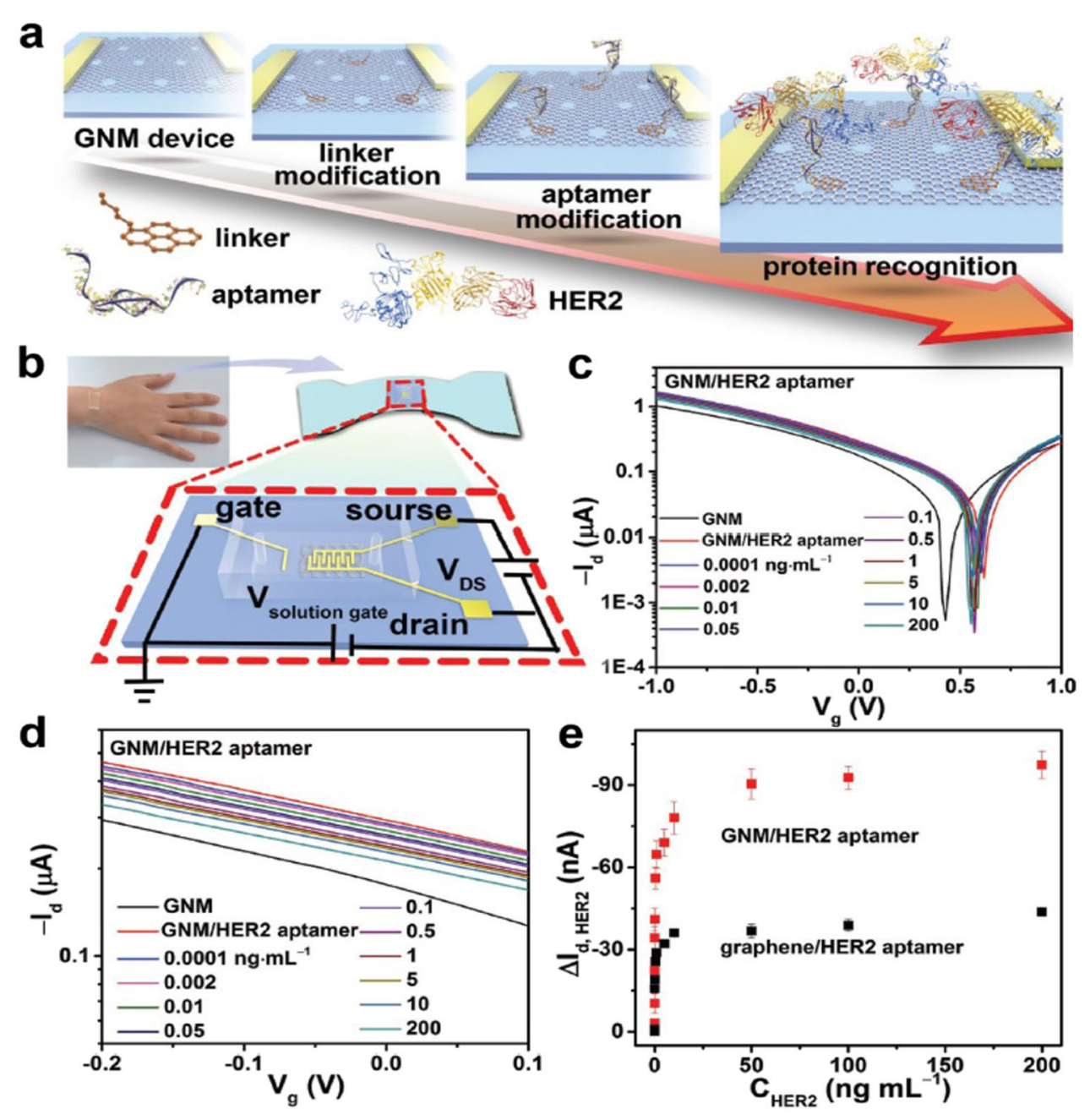

Fig. 8 GNM FET biosensor. a Schematic illustration of the fabrication of the GNM FET biosensor. $\mathbf{b}$ The GNM FET biosensor integrated on the PDMS film and attached on human skin. Enlarged view: corresponding schematic structure of the flexible biosensor. c Transfer characteristics of a solutiongated GNM FET biosensor in response to HER2 protein concentrations ranging from 0.0001 to $200 \mathrm{ng} \mathrm{ml}^{-1}$. The $V_{\mathrm{d}}$ is set at a fixed value of $-0.1 \mathrm{~V}$. d Magnified curve of (c) in the Vg range of -0.2 to $0.1 \mathrm{~V}$. e Current change ( $\triangle \mathrm{Id}$, HER2) versus HER2 concentration (CHER2). The error bars present the standard deviations of three measurements ${ }^{131}$. Adapted from ref. ${ }^{131}$, reproduced with permission (๑ 2017, WILEY-VCH Verlag GmbH \& Co. KGaA, Weinheim)

accessibility of electrolyte ions, while the $\mathrm{O}_{2}$-plasmatreated side showed the reverse relationship. Therefore, bending motions of the Janus sheet were triggered due to the different responses of each side towards the electrochemical charge/discharge process, and the degree of bending was tunable via the applied voltage across the Janus sheet, i.e., the modified graphene strip bent towards the hexane-plasma-treated side at negative bias, while bending towards the other side under a positive bias.

More recently, Mu et al. ${ }^{134}$ fabricated Janus-GO-based paper structures that could be used as walking devices through a self-folding motion. In that work, polydopamine (PDA)-grafted GO nanosheets were filtered through a pre-made rGO paper. In the filtration process,
GO-PDA was patterned using a filtration mask on rGO to yield a patterned GO-PDA/rGO bilayer. This Janus paper was then treated with hydroiodic acid, where GO-PDAcovered areas were protected from reduction and kept hydrophilic, and the uncovered parts were reduced and subsequently became hydrophobic. The hydrophilic GOPDA regions could readily absorb water and swell, and the swelling could be prohibited by heating through nearinfrared (NIR) exposure. Conversely, the hydrophobic rGO regions became insensitive to moisture change. Therefore, with the suitable design of a filtration mask and control of NIR exposure, the patterned GO-PDA/ rGO Janus paper could be programmed to bend/unbend, like a walking device and an artificial hand. 

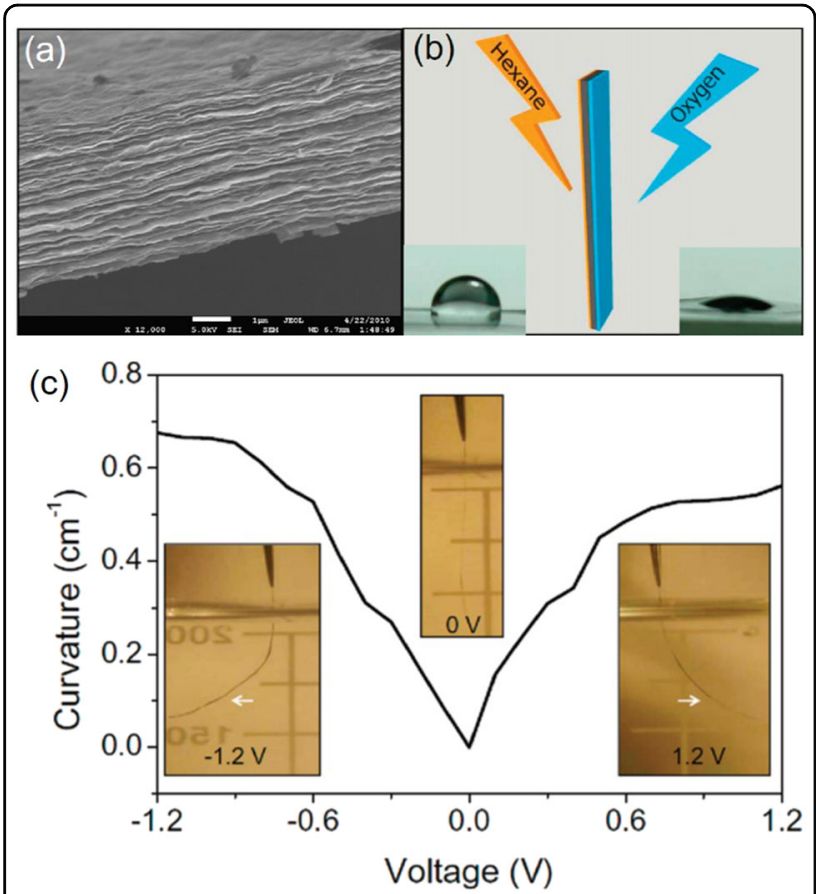

Fig. 9 Janus-graphene-based electrochemical actuator fabricated via direct plasma treatments. a Cross-section SEM image of a graphene film (scale bar: $1 \mu \mathrm{m}$ ). b Illustration of asymmetric plasma treatments of the graphene film using hexane and oxygen sources and the corresponding change in surface wettability. The water contact angles for the hexane-plasma (b, left inset) and oxygenplasma (b, right inset)-treated surfaces are $90^{\circ}$ and $15^{\circ}$, respectively. c Curvature change of an asymmetrically modified graphene strip as a function of applied CV potential within $\pm 1.2 \mathrm{~V}$. Insets show the status of the graphene strip at 1.2, 0 and $1.2 \mathrm{~V}^{133}$. Adapted from ref. ${ }^{133}$, reproduced with permission (๑ 2010, American Chemical Society)

\section{Lithium-sulfur batteries}

Lithium-sulfur ( $\mathrm{Li}-\mathrm{S})$ batteries employ relatively lowcost and abundant sulfur as the cathode material to hold a high theoretical energy density because of the conversion chemistry of the sulfur cathode. Therefore, Li-S has received great interest from the industry. However, Li-S batteries show the shuttle problem of polysulfide, i.e., the redox materials migrate out of the cathode and fix on the lithium anode in the solid form, which leads to serious capacity degradation and shortened cycling life of the battery. Thus, shuttle-inhibiting separators are always essential for long-lasting and effective $\mathrm{Li}-\mathrm{S}$ batteries. As mentioned above (section "Preparation of Janus graphene-based thin films"), a number of graphene-based thin films and membranes have been fabricated, and GO membranes are believed to be promising as an ion selective separator for $\mathrm{Li}-\mathrm{S}$ batteries because of its negatively charged nature, which should be permeable for lithium ions but reject anions ${ }^{135}$.

In 2015, Zhuang et al. ${ }^{136}$ reported a 3-layer separator for $\mathrm{Li}-\mathrm{S}$ batteries that was made up of a routine

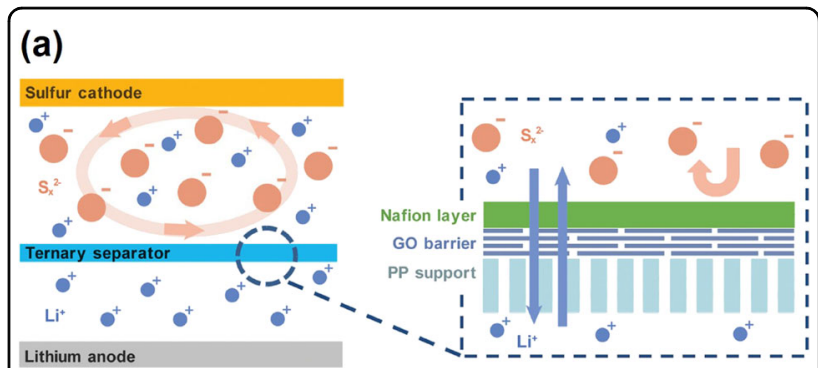

(b)

PP Separator

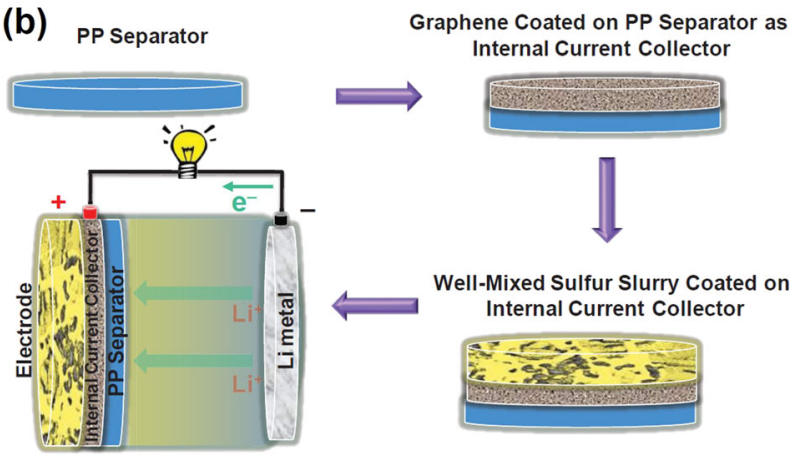

Fig. 10 Shuttle-inhibiting separators for Li-S batteries made up of Janus-graphene-based membranes. a Schematic of a ternary PP/ $\mathrm{GO} /$ Nafion separator: the PP layer serves as a freestanding continuous support. GO sheets not only cover the macropores on the PP separator with an ultralow loading of $0.0032 \mathrm{mg} \mathrm{cm}^{-2}$ but also serve as a physical barrier to selectively suppress the polysulfide anions. A Nafion layer of $0.050 \mathrm{mg} \mathrm{cm}^{-2}$ is coated on the $\mathrm{GO}$ to afford $-\mathrm{SO}^{3-}$ groups that allow ion hopping of positively charged species $\left(\mathrm{Li}^{+}\right)$but reject the hopping of negative ions of $\mathrm{S}_{n}{ }^{2-}$. Such a ternary-layered separator improves the Coulombic efficiency and capacity retention during the cycling of a Li-S battery ${ }^{136}$. Adapted from ref. ${ }^{136}$, reproduced with permission (@ 2015, WILEY-VCH Verlag GmbH \& Co. KGaA, Weinheim). b Schematic of the electrode configuration using an integrated structure of sulfur and G@PP separator and the corresponding battery assembly ${ }^{137}$. Adapted from ref. ${ }^{137}$, reproduced with permission (@ 2014, WILEY-VCH Verlag GmbH \& Co. KGaA, Weinheim)

polypropylene (PP) separator, a GO barrier layer and a dense ion-selective Nafion retarding layer ${ }^{136}$. In this work, the GO layer was first deposited on the porous PP membrane via vacuum filtration, and the Nafion layer was then coated on top. It was found that the ultrathin layer of GO (0.0032 $\mathrm{mg} \mathrm{cm}^{-2}, \sim 40$ layers) could pack tightly and effectively block the macropores of the PP matrix layer, whereas the uniform Nafion layer $\left(0.05 \mathrm{mg} \mathrm{cm}^{-2}\right)$ endowed one side of the separator with sulfonic acid groups, which further suppressed the crossover of polysulfide because of electrostatic repulsion. When this PP/ GO/Nafion separator was used to replace the PP separator in a $\mathrm{Li}-\mathrm{S}$ cell (with the Nafion side facing the sulfur electrode) (Fig. 10a), the initial capacity and Coulombic efficiency were enhanced from 969 to $1057 \mathrm{mAh} \mathrm{g}^{-1}$ and from $80 \%$ to over $95 \%$, respectively. The battery capacity 


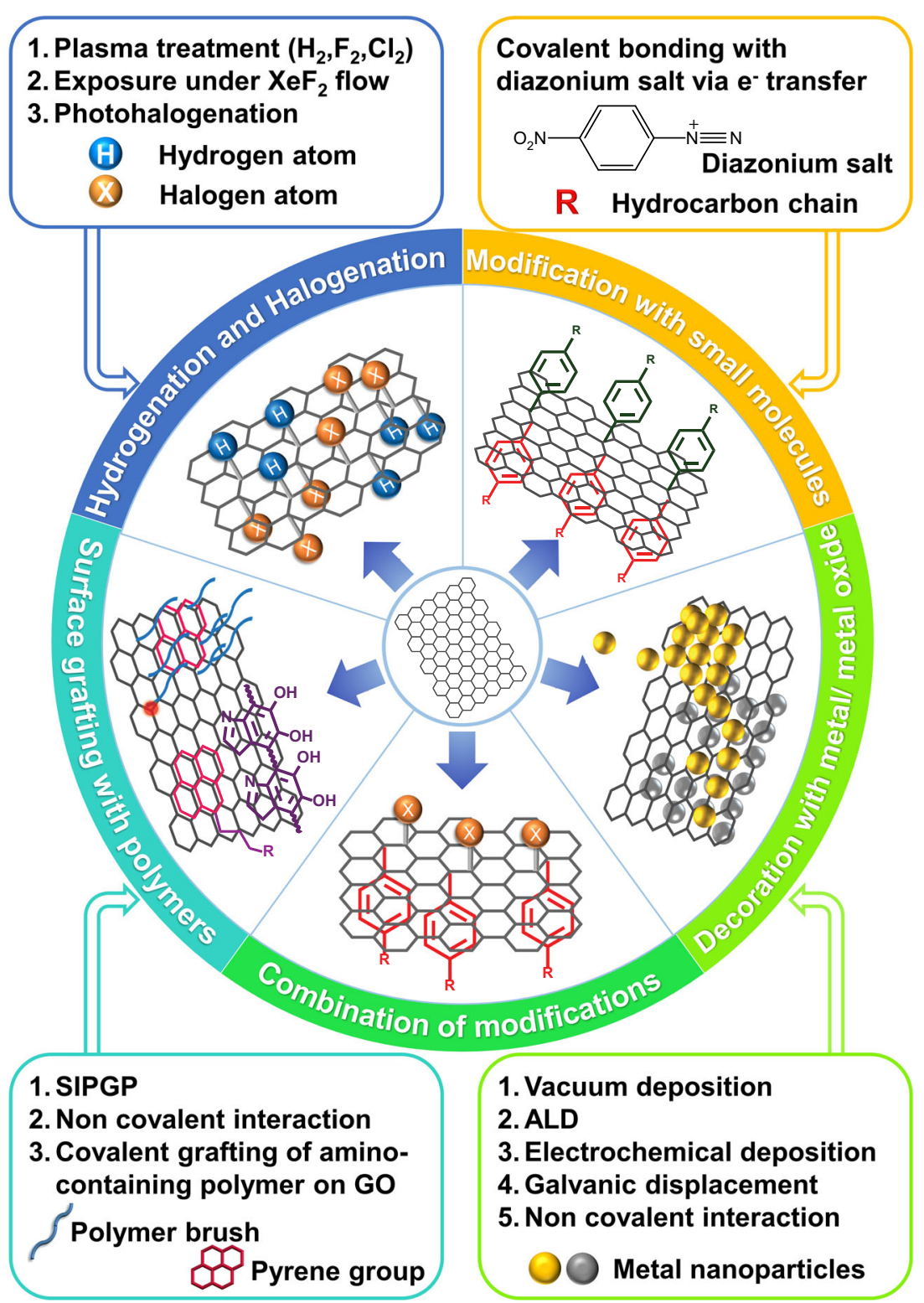

Scheme 1 Schematic diagram showing different common routes for fabricating 2D Janus graphene objects

degradation was also reduced from 0.34 to $0.18 \%$ per cycle (within 200 cycles).

Instead of a freestanding separator, as reported by Zhou et al., ${ }^{137}$ a flexible sulfur/graphene/PP separator integrated with the electrode was developed for $\mathrm{Li}-\mathrm{S}$ batteries. The fabrication process of this electrode was simply achieved by the slot coating of a layer of exfoliated graphene sheets and sulfur slurry on a commercial PP separator (Fig. 10b). In this structure, the graphene layer acted as a lightweight internal current collector that offered a continuous electron transfer pathway in between the separator and sulfur active material. It also acted as an effective barrier/reservoir for dissolved polysulfides to mitigate the shuttle effect. By adopting this S-G@PP as the cathode, long-cycle-life $\mathrm{Li}-\mathrm{S}$ batteries were achieved that obtained a capacity retention of $71.7 \%$ at $1.5 \mathrm{Ag}^{-1}$ with a very small capacity decay of $0.064 \%$ per cycle and a Coulombic efficiency approaching 100\% (after 500 cycles). These results were superior to the batteries fabricated using the conventional cathode structure (Al foil-S+PP separator) with a $31.9 \%$ capacity retention and a large capacity decay of $0.216 \%$ per cycle.

\section{Conclusion and perspective}

We have reviewed the fundamental theory, structural simulation, preparation and applications of Janus 
Table 1 Summary of general routes for fabricating 2D Janus graphene objects

\begin{tabular}{|c|c|c|c|c|}
\hline $\begin{array}{l}\text { Janus surface } \\
\text { modifications }\end{array}$ & Modifying agent & Functions & Methods for modification & Ref. \\
\hline \multirow[t]{4}{*}{$\begin{array}{l}\text { Hydrogenation and } \\
\text { halogenation }\end{array}$} & Hydrogen atoms & $\begin{array}{l}\text { Tuning bandgap of grapheme. Inducing } \\
\text { ferromagnetic properties. }\end{array}$ & Plasma treatment & 86 \\
\hline & \multirow[t]{3}{*}{$\begin{array}{l}\text { Halogen atoms }(\mathrm{F}, \mathrm{Cl} \\
\mathrm{Br} . .)\end{array}$} & \multirow[t]{2}{*}{$\begin{array}{l}\text { Tuning bandgap and surface wettability of } \\
\text { graphene. }\end{array}$} & $\begin{array}{l}\text { Exposure under } \mathrm{XeF}_{2} \text { flow } \\
\text { Plasma treatment }\end{array}$ & $\begin{array}{l}84,85 \\
86\end{array}$ \\
\hline & & & Photohalogenation & 87 \\
\hline & & Allowing cell adhesion. & Exposure under $\mathrm{XeF}_{2}$ flow & 126 \\
\hline \multirow{2}{*}{$\begin{array}{l}\text { Modification with small } \\
\text { molecules }\end{array}$} & Diazonium salt & \multirow{2}{*}{$\begin{array}{l}\text { Tuning bandgap/electrical properties of } \\
\text { graphene. }\end{array}$} & Covalent bonding & $92-95$ \\
\hline & F4-TCNQ & & Non-covalent interactions & 96 \\
\hline \multirow[t]{3}{*}{$\begin{array}{l}\text { Surface grafting with } \\
\text { polymers }\end{array}$} & \multirow[t]{3}{*}{$\begin{array}{l}\text { Polymer layers/ } \\
\text { polymer brushes }\end{array}$} & \multirow[t]{3}{*}{$\begin{array}{l}\text { Tuning surface wettability of grapheme. } \\
\text { Allowing immobilization of biomolecules.. }\end{array}$} & SIPGP & $\begin{array}{l}6,100 \\
122,123\end{array}$ \\
\hline & & & Covalent grafting on $\mathrm{GO}$ & 101 \\
\hline & & & Non-covalent interactions & 7,102 \\
\hline \multirow{6}{*}{$\begin{array}{l}\text { Deposition with metal/ } \\
\text { metal oxide }\end{array}$} & Metal layer & \multirow[t]{2}{*}{ Improving conductivity of graphene. } & Vacuum deposition & $109-111$ \\
\hline & \multirow[t]{3}{*}{ Metal NPs } & & Reduction of positive metal ions in & 115 \\
\hline & & $\begin{array}{l}\text { Allowing adsorption of aromatic molecules/ } \\
\text { biomolecules. }\end{array}$ & precursor solution & 117,119 \\
\hline & & $\begin{array}{l}\text { Enhancing the Raman signal of a specimen } \\
\text { when acting as a substrate. }\end{array}$ & & 116 \\
\hline & Metal oxide layer & $\begin{array}{l}\text { Pseudocapacitance materials for graphene- } \\
\text { based energy storage devices. }\end{array}$ & Atomic layer deposition & 113,114 \\
\hline & Metal oxide NPs/NRs & $\begin{array}{l}\text { Electron transport layer for graphene-based } \\
\text { photovoltaic electrodes. }\end{array}$ & $\begin{array}{l}\text { Electrochemical deposition } \\
\text { Non-covalent interaction on GO }\end{array}$ & $\begin{array}{l}124,132 \\
115\end{array}$ \\
\hline
\end{tabular}

graphene materials. The unique properties of various graphene-based Janus materials can be utilized to enhance and enrich the surface functionality and electronic performance of the much less reactive and functionalized pure graphene.

Scheme 1 and Table 1 summarize the strategies to prepare different kinds of Janus graphene and its derivatives, and Fig. 11 shows the development milestones of this field over the past two decades. From the chemical point of view, Janus graphene can be synthesized through the atomic replacement of the carbon atoms of graphene with hydrogen or halogen atoms or through the covalent grafting of graphene with functional ligands or polymers. Janus modification of graphene with hydrogen and halogens has proved to be an effective method for the band structure engineering of graphene, both theoretically and experimentally. Furthermore, through the grafting of small organic molecules and polymers on a graphene film, the desired surface reactivity towards biological and chemical substances can be tailored. Although these covalent modifications provide efficient transformation routes for graphene into desired functional states for a wide range of applications, they severely damage the conjugation of the basal plane, which sacrifices the major electronic merits of graphene. In contrast, non-covalent approaches allow the Janus modification of graphene without destroying its natural conjugated structure. Non-covalent modification typically makes use of the strong $\pi-\pi$ interaction between pyrene groups and graphene's basal plane or the nonselective binding ability of dopamine. Therefore, functional ligands or polymers are linked with the pyrene/ dopamine and then self-assembled onto the graphene surface. It should be noted that the research to date has mostly focused on the fundamental preparation and understanding of these materials. From the literature highlighted in this paper, the major applications of Janus graphene materials fall into two areas: (i) flexible and lightweight semiconducting/conducting materials for electronic applications and (ii) stimuli-driven actuators. Most of the applications demonstrated to date have been based on GO or rGO instead of single/few-layer graphene, in spite of their superior transparency, conductivity and 


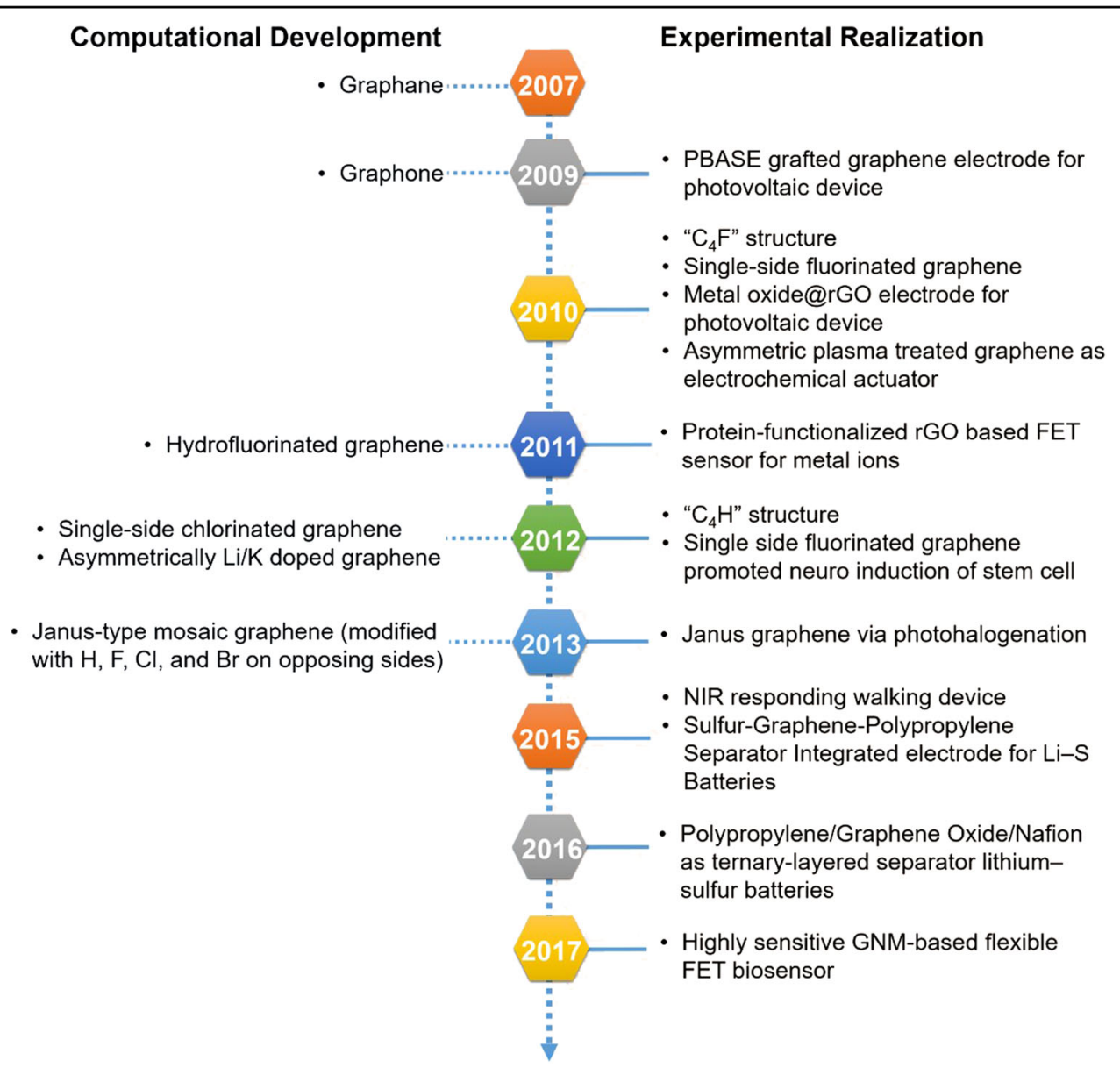

Fig. 11 Timeline of the development of graphene-based Janus 2D materials

few-atom thickness. Furthermore, most of these techniques are difficult to scale-up. One major reason is the relatively high production cost for producing large-area and high-quality pristine graphene films via epitaxial growth or CVD processes; special handling skills, costly techniques and machines are also needed for certain modifications of ultrathin carbon-based materials. For example, the halogenation of graphene is an energyconsuming (high-temperature) process, and a polymeric protection layer is always needed for transferring the graphene layer in most Janus graphene fabrication process. Any unremoved polymeric substance could contaminate the graphene samples, negatively impacting the reactivity and electronic performance of the modified Janus graphene. Hence, extra comprehensive rinsing steps are required.

To conclude, the rational design of graphene-based Janus materials with different shapes, sizes and compositions can be achieved via the asymmetric surface functionalization of target graphene derivatives. In principle, this could significantly extend the use of graphene to a wide range of applications, e.g., surface modification, sensing, electronics, biology, medicine, actuators, etc. The research in this field is still in the early stage, mostly focusing on the preparation of various Janus graphene materials and the understanding of their properties. To realize real-world applications, more efficient and lowcost Janus graphene fabrication strategies should be developed in the future. Meanwhile, commercialization also requires a deepened understanding of a number of phenomena: (i) the feasibilities and effects of attaching different atoms onto the basal plane of graphene in nondestructive ways, (ii) the influence of the surface-grafted atoms/molecules on the bandgap structure and physical properties of graphene, and (iii) the stability of different anchored functional groups or particles on graphene.

\section{Acknowledgements}

We acknowledge General Research Fund of Hong Kong (PolyU 5036/13P, PolyU 153041/14P) and The Hong Kong Polytechnic University (Project 1ZVGH) for financial support of the work.

Conflict of interest

The authors declare that they have no conflict of interest. 


\section{Publisher's note}

Springer Nature remains neutral with regard to jurisdictional claims in

published maps and institutional affiliations.

Received: 14 October 2017 Revised: 21 December 2017 Accepted: 4 February 2018.

Published online: 12 April 2018

\section{References}

1. Gagarin, M. Ancient Greece and Rome (Oxford University Press, New York, 2009).

2. Casagrande, C., Fabre, P., Raphaël, E. \& Veyssié, M. "Janus beads": realization and behaviour at water/oil interfaces. EPL (Europhys. Lett.) 9, 251 (1989).

3. Yang, H.-C., Hou, J., Chen, V. \& Xu, Z.-K. Janus membranes: exploring duality for advanced separation. Angew. Chem. Int. Ed. 55, 13398-13407 (2016)

4. Pang, X., Wan, C., Wang, M. \& Lin, Z. Strictly biphasic soft and hard Janus structures: synthesis, properties, and applications. Angew. Chem. Int. Ed. 53, 5524-5538 (2014).

5. Xiao, P. et al. Integration of a patterned conductive carbon nanotube thin film with an insulating hydrophobic polymer carpet into robust 2D Janus hybrid flexible electronics. J. Mater. Chem. C 4, 9750-9755 (2016).

6. Xiao, P. et al. 2D Janus hybrid materials of polymer-grafted carbon nanotube/graphene oxide thin film as flexible, miniature electric carpet. Adv. Funct. Mater. 25, 2428-2435 (2015).

7. Wang, Y., Chen, X., Zhong, Y., Zhu, F. \& Loh, K. P. Large area, continuous, fewlayered graphene as anodes in organic photovoltaic devices. Appl. Phys. Lett. 95, 063302 (2009).

8. Zhang, L. et al. Macroscopic ultrathin film as bio-inspired interfacial reactor for fabricating 2D freestanding Janus CNTs/AuNPs hybrid nanosheets with enhanced electrical performance. Adv. Mater. Interfaces 3, 1600170-n/a (2016).

9. Yi, Y., Sanchez, L., Gao, Y. \& Yu, Y. Janus particles for biological imaging and sensing. Analyst 141, 3526-3539 (2016).

10. Schick, I. et al. Inorganic Janus particles for biomedical applications. Beilstein J. Nanotechnol. 5, 2346-2362 (2014).

11. Wang, Z., Wang, Y. \& Liu, G. Rapid and efficient separation of oil from oil-inwater emulsions using a Janus cotton fabric. Angew. Chem. Int. Ed. 55, 1291-1294 (2016).

12. Gu, J. et al. Janus polymer/carbon nanotube hybrid membranes for oil/water separation. ACS Appl. Mater. Interfaces 6, 16204-16209 (2014).

13. Kirillova, A. et al. Platelet Janus particles with hairy polymer shells for multifunctionalmaterials. ACS Appl. Mater. Interfaces 6, 13106-13114 (2014).

14. Abraham, S., Ma, G. \& Montemagno, C. D. Janus carbon nanotube membranes by selective surface plasmoxidation. Adv. Mater. Interfaces 3 , 1600445-n/a (2016).

15. Zhao, L. B. et al. Generation of Janus alginate hydrogel particles with magnetic anisotropy for cell encapsulation. Lab. Chip. 9, 2981-2986 (2009).

16. Xie, H., She, Z.-G., Wang, S., Sharma, G. \& Smith, J. W. One-step fabrication of polymeric Janus nanoparticles for drug delivery. Langmuir 28, 4459-4463 (2012).

17. Walther, A. \& Müller, A. H. E. Janus particles: synthesis, self-assembly, physical properties, and applications. Chem. Rev. 113, 5194-5261 (2013).

18. Cheng, Q. et al. Janus interface materials: superhydrophobic air/solid interface and superoleophobic water/solid interface inspired by a lotus leaf. Soft Matter 7, 5948-5951 (2011).

19. Yue, B. et al. "Lotus-effect" tape: imparting superhydrophobicity to solid materials with an electrospun Janus composite mat. RSC Adv. 6, 17215-17221 (2016).

20. Liang, F., Zhang, C. \& Yang, Z. Rational design and synthesis of Janus composites. Adv. Mater. 26, 6944-6949 (2014).

21. Synytska, A. \& Ionov, L. Stimuli-responsive Janus particles. Part. Part. Syst. Charact. 30, 922-930 (2013).

22. Gao, L., Zhang, K. \& Chen, Y. Dumpling-like nanocomplexes of foldable Janus polymer sheets and spheres. ACS Macro Lett. 1, 1143-1145 (2012).

23. Zheng, $Z$. et al. Janus nanomembranes: a generic platform for chemistry in two dimensions. Angew. Chem. Int. Ed. 49, 8493-8497 (2010).
24. Zhang, H., Hao, R., Jackson, J. K., Chiao, M. \& Yu, H. Janus ultrathin film from multi-level self-assembly at air-water interfaces. Chem. Commun. 50, 14843-14846 (2014).

25. Dorvee, J. R., Derfus, A. M., Bhatia, S. N. \& Sailor, M. J. Manipulation of liquid droplets using amphiphilic, magnetic one-dimensional photonic crystal chaperones. Nat. Mater. 3, 896-899 (2004).

26. Liang, F. et al. Inorganic Janus nanosheets. Angew. Chem. Int. Ed. 50, 2379-2382 (2011).

27. Yang, $\mathrm{H}$. et al. Responsive Janus composite nanosheets. Macromolecules 46 2754-2759 (2013).

28. Liu, J., Liu, G., Zhang, M., Sun, P. \& Zhao, H. Synthesis and self-assembly of amphiphilic Janus laponite disks. Macromolecules 46, 5974-5984 (2013).

29. Liu, Y., Liang, F., Wang, Q., Qu, X. \& Yang, Z. Flexible responsive Janus nanosheets. Chem. Commun. 51, 3562-3565 (2015).

30. Sheng, L., Chen, H., Fu, W. \& Li, Z. Janus silica hollow spheres prepared via interfacial biosilicification. Langmuir 31, 11964-11970 (2015).

31. Geim, A. K. \& Novoselov, K. S. The rise of graphene. Nat. Mater. 6, 183-191 (2007).

32. Lee, C., Wei, X., Kysar, J. W. \& Hone, J. Measurement of the elastic properties and intrinsic strength of monolayer graphene. Science 321, 385-388 (2008).

33. Li, X. et al. Large-area synthesis of high-quality and uniform graphene films on copper foils. Science 324, 1312 (2009).

34. Obraztsov, A. N. Chemical vapour deposition: making graphene on a large scale. Nat. Nano 4, 212-213 (2009).

35. Reina, A. et al. Large area, few-layer graphene films on arbitrary substrates by chemical vapor deposition. Nano. Lett. 9, 30-35 (2009).

36. Vallés, $\mathrm{C}$. et al. Solutions of negatively charged graphene sheets and ribbons. J. Am. Chem. Soc. 130, 15802-15804 (2008).

37. Li, X. et al. Highly conducting graphene sheets and Langmuir-Blodgett films. Nat. Nano 3, 538-542 (2008).

38. Yang, $\mathrm{H}$. et al. Graphene barristor, a triode device with a gate-controlled Schottky barrier. Science 336, 1140 (2012).

39. Yoo, E. et al. Large reversible Li storage of graphene nanosheet families for use in rechargeable lithium ion batteries. Nano. Lett. 8, 2277-2282 (2008).

40. Yang, S., Feng, X., Ivanovici, S. \& Müllen, K. Fabrication of grapheneencapsulated oxide nanoparticles: towards high-performance anode materials for lithium storage. Angew. Chem. Int. Ed. 49, 8408-8411 (2010).

41. Wang, $\mathrm{H}$. et al. Mn3O4-graphene hybrid as a high-capacity anode material for lithium ion batteries. J. Am. Chem. Soc. 132, 13978-13980 (2010).

42. Huang, $Y$., Liang, J. \& Chen, Y. An overview of the applications of graphenebased materials in supercapacitors. Small 8, 1805-1834 (2012).

43. Zhang, B., Yu, B., Zhou, F. \& Liu, W. Polymer brush stabilized amorphous $\mathrm{MnO} 2$ on graphene oxide sheets as novel electrode materials for high performance supercapacitors. J. Mater. Chem. A 1, 8587-8592 (2013).

44. Peng, L., Zhu, Y., Chen, D., Ruoff, R. S. \& Yu, G. Two-Dimensional materials for beyond-lithium-ion batteries. Adv. Energy Mater. 6, 1600025-n/a (2016).

45. Li, J., Niu, L., Zheng, Z. \& Yan, F. Photosensitive graphene transistors. Adv. Mater. 26, 5239-5273 (2014).

46. Chang, $\mathrm{H}$. et al. Thin film field-effect phototransistors from bandgap-tunable, solution-processed, few-layer reduced graphene oxide films. Adv. Mater. 22, 4872-4876 (2010)

47. Lee, J., Tao, L., Hao, Y., Ruoff, R. S. \& Akinwande, D. Embedded-gate graphene transistors for high-mobility detachable flexible nanoelectronics. Appl. Phys. Lett. 100, 152104 (2012).

48. Gomez De Arco, L. et al. Continuous, highly flexible, and transparent graphene films by chemical vapor deposition for organic photovoltaics. ACS Nano 4, 2865-2873 (2010).

49. Lee, J.-S., Ahn, H.-J., Yoon, J.-C. \& Jang, J.-H. Three-dimensional nano-foam of few-layer graphene grown by CVD for DSSC. Phys. Chem. Chem. Phys. 14, 7938-7943 (2012).

50. Wang, X., Zhi, L. \& Müllen, K. Transparent, conductive graphene electrodes for dye-sensitized solar cells. Nano. Lett. 8, 323-327 (2008).

51. Wang, $X$. et al. Transparent carbon films as electrodes in organic solar cells. Angew. Chem. Int. Ed. 47, 2990-2992 (2008).

52. Yan, J., Ye, Q., Wang, X., Yu, B. \& Zhou, F. CdS/CdSe quantum dot cosensitized graphene nanocomposites via polymer brush templated synthesis for potential photovoltaic applications. Nanoscale 4, 2109-2116 (2012).

53. Huang, X., Zeng, Z., Fan, Z., Liu, J. \& Zhang, H. Graphene-based electrodes. Adv. Mater. 24, 5979-6004 (2012).

54. Sim, U. et al. N-doped monolayer graphene catalyst on silicon photocathode for hydrogen production. Energy Environ. Sci. 6, 3658-3664 (2013). 
55. Li, Y. et al. MoS2 nanoparticles grown on graphene: an advanced catalyst for the hydrogen evolution reaction. J. Am. Chem. Soc. 133, 7296-7299 (2011).

56. Schedin, F. et al. Detection of individual gas molecules adsorbed on graphene. Nat. Mater. 6, 652-655 (2007).

57. Hong, J.-Y. \& Jang, J. Micropatterning of graphene sheets: recent advances in techniques and applications. J. Mater. Chem. 22, 8179-8191 (2012).

58. Shao, Y. et al. Graphene based electrochemical sensors and biosensors: a review. Electroanalysis 22, 1027-1036 (2010).

59. Marchesan, S., Ballerini, L. \& Prato, M. Nanomaterials for stimulating nerve growth. Science 356, 1010 (2017).

60. Edwards, R. S. \& Coleman, K. S. Graphene film growth on polycrystalline metals. Acc. Chem. Res. 46, 23-30 (2013).

61. Wu, S., He, Q., Tan, C., Wang, Y. \& Zhang, H. Graphene-based electrochemical sensors. Small 9, 1160-1172 (2013).

62. Huang, X., Qi, X., Boey, F. \& Zhang, H. Graphene-based composites. Chem. Soc. Rev. 41, 666-686 (2012).

63. Georgakilas, V. et al. Functionalization of graphene: covalent and noncovalent approaches, derivatives and applications. Chem. Rev. 112 6156-6214 (2012).

64. Bonaccorso, F. et al. Graphene, related two-dimensional crystals, and hybrid systems for energy conversion and storage. Science 347, 1246501 (2015)

65. Sofo, J. O., Chaudhari, A. S. \& Barber, G. D. Graphane: a two-dimensional hydrocarbon. Phys. Rev. B 75, 153401 (2007).

66. Zhou, J. et al. Ferromagnetism in semihydrogenated graphene sheet. Nano. Lett. 9, 3867-3870 (2009).

67. Xiang, H. J., Kan, E. J., Wei, S.-H., Gong, X. G. \& Whangbo, M. H. Thermodynamically stable single-side hydrogenated graphene. Phys. Rev. B 82, 165425 (2010).

68. Haberer, D. et al. Evidence for a new two-dimensional C4H-type polymer based on hydrogenated graphene. Adv. Mater. 23, 4497-4503 (2011).

69. Li, Y. \& Chen, Z. Patterned partially hydrogenated graphene $(\mathrm{C} 4 \mathrm{H})$ and its one-dimensional analogues: a computational study. J. Phys. Chem. C 116, 4526-4534 (2012).

70. Bahr, J. L. et al. Functionalization of carbon nanotubes by electrochemical reduction of aryl diazonium salts: a bucky paper electrode. J. Am. Chem. Soc. 123, 6536-6542 (2001).

71. Yang, M., Zhou, L., Wang, J., Liu, Z. \& Liu, Z. Evolutionary chlorination of graphene: from charge-transfer complex to covalent bonding and nonbonding. J. Phys. Chem. C 116, 844-850 (2012).

72. Singh, R. \& Bester, G. Hydrofluorinated graphene: two-dimensional analog of polyvinylidene fluoride. Phys. Rev. B 84, 155427 (2011).

73. Li, F. \& Li, Y. Band gap modulation of Janus graphene nanosheets by interlayer hydrogen bonding and the external electric field: a computational study. J. Mater. Chem. C 3, 3416-3421 (2015).

74. Yang, M. et al. Bandgap opening in Janus-type mosaic graphene. J. Appl. Phys. 113, 084313 (2013).

75. Ong, M. T. \& Reed, E. J. Engineered piezoelectricity in graphene. ACS Nano 6, 1387-1394 (2012).

76. Ong, M. T., Duerloo, K.-A. N. \& Reed, E. J. The effect of hydrogen and fluorine coadsorption on the piezoelectric properties of graphene. J. Phys. Chem. C 117, 3615-3620 (2013)

77. Kim, H. J., Noor-A-Alam, M., Son, J. Y. \& Shin, Y.-H. Origin of piezoelectricity in monolayer halogenated graphane piezoelectrics. Chem. Phys. Lett. 603, 62-66 (2014).

78. Yuan, L., Li, Z., Yang, J. \& Hou, J. G. Diamondization of chemically functionalized graphene and graphene-BN bilayers. Phys. Chem. Chem. Phys. 14, 8179-8184 (2012).

79. Barboza, A. P. et al. Room-temperature compression-induced diamondization of few-layer graphene. Adv. Mater. 23, 3014-3017 (2011).

80. Huang, X. et al. Graphene-based materials: synthesis, characterization, properties, and applications. Small 7, 1876-1902 (2011).

81. Chen, D., Feng, H. \& Li, J. Graphene oxide: preparation, functionalization, and electrochemical applications. Chem. Rev. 112, 6027-6053 (2012).

82. Mattevi, C., Kim, H. \& Chhowalla, M. A review of chemical vapour deposition of graphene on copper. J. Mater. Chem. 21, 3324-3334 (2011).

83. Park, S. \& Ruoff, R. S. Chemical methods for the production of graphenes. Nat. Nano 4, 217-224 (2009).

84. Robinson, J. T. et al. Properties of fluorinated graphene films. Nano. Lett. 10, 3001-3005 (2010).
85. Nair, R. R. et al. Fluorographene: a two-dimensional counterpart of teflon. Small 6, 2877-2884 (2010)

86. $\mathrm{Wu}, \mathrm{J}$. et al. Controlled chlorine plasma reaction for noninvasive graphene doping. J. Am. Chem. Soc. 133, 19668-19671 (2011).

87. Zhang, L. et al. Janus graphene from asymmetric two-dimensional chemistry. Nat. Commun. 4, 1443 (2013).

88. Delamar, M., Hitmi, R., Pinson, J. \& Saveant, J. M. Covalent modification of carbon surfaces by grafting of functionalized aryl radicals produced from electrochemical reduction of diazonium salts. J. Am. Chem. Soc. 114 5883-5884 (1992).

89. Liu, Y.-C. \& McCreery, R. L. Reactions of organic monolayers on carbon surfaces observed with unenhanced Raman spectroscopy. J. Am. Chem. Soc. 117, 11254-11259 (1995).

90. Kamaras, K., Itkis, M. E., Hu, H., Zhao, B. \& Haddon, R. C. Covalent bond formation to a carbon nanotube metal. Science 301, 1501 (2003).

91. Bahr, J. L. et al. Functionalization of carbon nanotubes by electrochemical reduction of aryl diazonium salts: a bucky paper electrode. J. Am. Chem. Soc. 123, 6536-6542 (2001).

92. Bekyarova, E. et al. Chemical modification of epitaxial graphene: spontaneous grafting of aryl groups. J. Am. Chem. Soc. 131, 1336-1337 (2009).

93. Sinitskii, A. et al. Kinetics of diazonium functionalization of chemically converted graphene nanoribbons. ACS Nano 4, 1949-1954 (2010).

94. Farmer, D. B. et al. Chemical doping and electron-hole conduction asymmetry in graphene devices. Nano. Lett. 9, 388-392 (2009).

95. Bissett, M. A., Takesaki, Y., Tsuji, M. \& Ago, H. Increased chemical reactivity achieved by asymmetrical 'Janus' functionalisation of graphene. RSC Adv. 4 52215-52219 (2014).

96. Coletti, $C$. et al. Charge neutrality and band-gap tuning of epitaxial graphene on SiC by molecular doping. Phys. Rev. B 81, 235401 (2010).

97. Stachowiak, T. B., Svec, F. \& Fréchet, J. M. J. Patternable protein resistant surfaces for multifunctional microfluidic devices via surface hydrophilization of porous polymer monoliths using photografting. Chem. Mater. 18, 5950-5957 (2006)

98. Deng, J. P., Yang, W. T. \& Ranby, B. Auto-initiating performance of styrene on surface photografting polymerization. Macromol. Rapid Commun. 22, 535-538 (2001).

99. Steenackers, M., Kuller, A., Stoycheva, S., Grunze, M. \& Jordan, R. Structured and gradient polymer brushes from biphenylthiol self-assembled monolayers by self-initiated photografting and photopolymerization (SIPGP). Langmuir 25, 2225-2231 (2009).

100. Steenackers, M. et al. Polymer brushes on graphene. J. Am. Chem. Soc. 133, 10490-10498 (2011).

101. Wu, H., Yi, W., Chen, Z., Wang, H. \& Du, Q. Janus graphene oxide nanosheets prepared via Pickering emulsion template. Carbon N. Y. 93, 473-483 (2015).

102. Xiao, P. et al. A microcontact printing induced supramolecular self-assembled photoactive surface for patterning polymer brushes. Chem. Commun. 49 , 11167-11169 (2013).

103. Gao, T. et al. Transferable, transparent and functional polymer@graphene 2D objects. NPG Asia Mater. 6, e130 (2014).

104. Gao, T. et al. Noncovalent microcontact printing for grafting patterned polymer brushes on graphene films. Langmuir 29, 1054-1060 (2013).

105. Xu, Y., Bai, H., Lu, G., Li, C. \& Shi, G. Flexible graphene films via the filtration of water-soluble noncovalent functionalized graphene sheets. J. Am. Chem. Soc. 130, 5856-5857 (2008)

106. Yang, Y. et al. Preparation of Janus graphene oxide (GO) nanosheets based on electrostatic assembly of GO nanosheets and polystyrene microspheres. Macromol. Rapid Commun. 37, 1520-1526 (2016).

107. Kim, B. H. et al. Mussel-inspired block copolymer lithography for low surface energy materials of teflon, graphene, and gold. Adv. Mater. 23, 5618-5622 (2011).

108. Seokhan, P. et al. Device-oriented graphene nanopatterning by musselinspired directed block copolymer self-assembly. Nanotechnology 25, 014008 (2014).

109. Ren, Y. et al. Controlling the electrical transport properties of graphene by in situ metal deposition. Appl. Phys. Lett. 97, 053107 (2010).

110. Zhou, $\mathrm{H}$. et al. Thickness-dependent morphologies of gold on N-layer graphenes. J. Am. Chem. Soc. 132, 944-946 (2010).

111. Li, Y. et al. Janus graphene hybrids: $2 \mathrm{D}$ monodispersed gold nanoarrays on graphene with controlled structure and high stability. in 2014 International Conference on Manipulation, Manufacturing and Measurement on the Nanoscale (3M-NANO). 117-121 (2014). 
112. Sun, S. et al. Single-atom catalysis using Pt/graphene achieved through atomic layer deposition. Sci. Rep. 3, 1775 (2013).

113. Wang, X., Tabakman, S. M. \& Dai, H. Atomic layer deposition of metal oxides on pristine and functionalized graphene. J. Am. Chem. Soc. 130, 8152-8153 (2008).

114. George, S. M. Atomic layer deposition: an overview. Chem. Rev. 110, 111-131 (2010).

115. Yu, D., Nagelli, E., Naik, R. \& Dai, L. Asymmetrically functionalized graphene for photodependent diode rectifying behavior. Angew. Chem. Int. Ed. 50, 6575-6578 (2011).

116. Lu, G. et al. Surface enhanced Raman scattering of Ag or Au nanoparticledecorated reduced graphene oxide for detection of aromatic molecules. Chem. Sci. 2, 1817-1821 (2011).

117. Yin, Z. et al. Real-time DNA detection using Pt nanoparticle-decorated reduced graphene oxide field-effect transistors. Nanoscale 4, 293-297 (2012).

118. Toth, P. S., Velický, M., Ramasse, Q. M., Kepaptsoglou, D. M. \& Dryfe, R. A. W. Symmetric and asymmetric decoration of graphene: bimetal-graphene sandwiches. Adv. Funct. Mater. 25, 2899-2909 (2015).

119. Gutes, A. et al. Graphene decoration with metal nanoparticles: towards easy integration for sensing applications. Nanoscale 4, 438-440 (2012).

120. Compton, O. C. \& Nguyen, S. T. Graphene oxide, highly reduced graphene oxide, and graphene: versatile building blocks for carbon-based materials. Small 6, 711-723 (2010).

121. Zhu, Y. et al. Graphene and graphene oxide: synthesis, properties, and applications. Adv. Mater. 22, 3906-3924 (2010).

122. Xiao, P. et al. Micro-contact printing of graphene oxide nanosheets for fabricating patterned polymer brushes. Chem. Commun. 50, 7103-7106 (2014).

123. Han, D. et al. Polymer brush functionalized Janus graphene oxide/chitosan hybrid membranes. RSC Adv. 4, 22759-22762 (2014).

124. Wu, S. et al. Electrochemical deposition of semiconductor oxides on reduced graphene oxide-based flexible, transparent, and conductive electrodes. J. Phys. Chem. C 114, 11816-11821 (2010).
125. McGrail, B. T. et al. Selective mono-facial modification of graphene oxide nanosheets in suspension. Chem. Commun. 52, 288-291 (2016).

126. Wang, $Y$. et al. Fluorinated graphene for promoting neuro-induction of stem cells. Adv. Mater. 24, 4285-4290 (2012).

127. Singh, $M$. et al. Noncovalently functionalized monolayer graphene for sensitivity enhancement of surface plasmon resonance immunosensors. J. Am. Chem. Soc. 137, 2800-2803 (2015).

128. Liu, G., Ahsan, S., Khitun, A. G., Lake, R. K. \& Balandin, A. A. Graphene-based non-Boolean logic circuits. J. Appl. Phys. 114, 154310 (2013).

129. Sarkar, S. et al. Organometallic hexahapto functionalization of single layer graphene as a route to high mobility graphene devices. Adv. Mater. 25, 1131-1136 (2013).

130. Sudibya, H. G., He, Q., Zhang, H. \& Chen, P. Electrical detection of metal ions using field-effect transistors based on micropatterned reduced graphene oxide films. ACS Nano 5, 1990-1994 (2011).

131. Yang, Y. et al. Ultrafine graphene nanomesh with large on/off ratio for highperformance flexible biosensors. Adv. Funct. Mater. 27, 1604096-n/a (2017).

132. Yin, Z. et al. Electrochemical deposition of $\mathrm{ZnO}$ nanorods on transparent reduced graphene oxide electrodes for hybrid solar cells. Small 6, 307-312 (2010).

133. Xie, X. et al. An asymmetrically surface-modified graphene film electrochemical actuator. ACS Nano 4, 6050-6054 (2010).

134. $\mathrm{Mu}, \mathrm{J}$. et al. Origami-inspired active graphene-based paper for programmable instant self-folding walking devices. Sci. Adv. 1, e1500533 (2015).

135. Peng, H. J. et al. Janus separator of polypropylene-supported cellular graphene framework for sulfur cathodes with high utilization in lithium-sulfur batteries. Adv. Sci. 3, 1500268 (2016).

136. Zhuang, T. Z. et al. Rational integration of polypropylene/graphene oxide/ nafion as ternary-layered separator to retard the shuttle of polysulfides for lithium-sulfur batteries. Small 12, 381-389 (2016).

137. Zhou, G. et al. A flexible sulfur-graphene-polypropylene separator integrated electrode for advanced Li-S batteries. Adv. Mater. 27, 641-647 (2015). 